\title{
Task-Demand-Dependent Neural Representation of Odor Information in the Olfactory Bulb and Posterior Piriform Cortex
}

\author{
Dㅣㄹ. \\ and ${ }^{\circ}$ Anan Li \\ Jiangsu Key Laboratory of Brain Disease and Bioinformation, Research Center for Biochemistry and Molecular Biology, Xuzhou Medical University, \\ Xuzhou, 221004, China
}

In awake rodents, the neural representation of olfactory information in the olfactory bulb is largely dependent on brain state and behavioral context. Learning-modified neural plasticity has been observed in mitral/tufted cells, the main output neurons of the olfactory bulb. Here, we propose that the odor information encoded by mitral/tufted cell responses in awake mice is highly dependent on the behavioral task demands. We used fiber photometry to record calcium signals from the mitral/tufted cell population in awake, head-fixed male mice under different task demands. We found that the mitral/tufted cell population showed similar responses to two distinct odors when the odors were presented in the context of a go/go task, in which the mice received a water reward regardless of the identity of the odor presented. However, when the same odors were presented in a go/no-go task, in which one odor was rewarded and the other was not, then the mitral cell population responded very differently to the two odors, characterized by a robust reduction in the response to the nonrewarded odor. Thus, the representation of odors in the mitral/tufted cell population depends on whether the task requires discrimination of the odors. Strikingly, downstream of the olfactory bulb, pyramidal neurons in the posterior piriform cortex also displayed a task-demand-dependent neural representation of odors, but the anterior piriform cortex did not, indicating that these two important higher olfactory centers use different strategies for neural representation.

Key words: electrophysiology; fiber photometry; olfactory bulb; piriform cortex; task

Significance Statement

The most important task of the olfactory system is to generate a precise representation of odor information under different brain states. Whether the representation of odors by neurons in olfactory centers such as the olfactory bulb and the piriform cortex depends on task demands remains elusive. We find that odor representation in the mitral/tufted cells of the olfactory bulb depends on whether the task requires odor discrimination. A similar neural representation is found in the posterior piriform cortex but not the anterior piriform cortex, indicating that these higher olfactory centers use different representational strategies. The taskdemand-dependent representational strategy is likely important for facilitating information processing in higher brain centers responsible for decision making and encoding of salience.

\section{Introduction}

Sensory systems have the ability to perceive the dynamic external environment precisely and to facilitate behavior toward the most

Received May 28, 2019; revised Oct. 16, 2019; accepted 0ct. 19, 2019.

Author contributions: D.W. and A.L. designed research; D.W., P.L., X.M., Z.Z., T.C., J.X., and C.S. performed research; D.W., P.L., X.M., and A.L. analyzed data; D.W. and A.L. wrote the paper.

This work was supported by the National Natural Science Foundation of China (31571082 and 31872771 to A.L., 31700895 to D.W., and 31700896 to C.S.), Priority Academic Program Development of Jiangsu Higher Education Institutions (16KJA180007 to A.L.), and Natural Science Foundation of Jiangsu Province (BK20170260 to D.W. and BK20170254 to C.S.). We thank Yang Zhou, Han Xu, and Siqi Jing for technical assistance; Diego Restrepo for critical comments; and Shuming Duan from Zhejiang University for kindly providing the Thy1-Cre mice.

The authors declare no competing financial interests. important stimuli. To accomplish this complex task, the brain must filter sensory information, using selective attention to focus on salient stimuli (Berridge and Aldridge, 2008; Carlson et al., 2018). The brain can also respond variably to a specific stimulus presented on multiple trials, depending on the task demands and the behavioral states of the animal (Fontanini and Katz, 2008; Otazu et al., 2009; Gutnisky et al., 2017; Li et al., 2019). For instance, mice will focus on an odor by sniffing quickly, then

*D.W., P.L., and X.M. contributed equally to this work.

Correspondence should be addressed to Anan Li at anan.li@xzhmu.edu.cn.

https://doi.org/10.1523/JNEUROSCI.1234-19.2019

Copyright $\odot 2019$ the authors 
make a careful sensory decision and move toward the odor if it is associated with food. However, mice will neglect the same odor if it is related neither to a reward nor to a potential danger. Thus, odor detection and odor discrimination are important task demands for animals. Whether the brain discriminates odors may depend on the task demands, and the neural representation of the same odor under different task demands may differ.

As the first processing center and a crucial hub of the olfactory system, the olfactory bulb (OB) plays an important role in odor detection and discrimination (Gire et al., 2013b; Uchida et al., 2014; Wilson et al., 2017; Chong and Rinberg, 2018; Li et al., 2019). In the OB, the mitral/tufted cells (M/Ts), which are main output neurons, receive direct excitatory input from olfactory sensory neurons at the glomeruli and send the neural information to higher olfactory centers, including the anterior olfactory nucleus and the piriform cortex (Uchida et al., 2014; Vaaga and Westbrook, 2016). M/T activity is modified by dynamic interactions with GABAergic and dopaminergic interneurons within the OB (Burton, 2017; Vaaga et al., 2017), and circuit activity in the $\mathrm{OB}$ is modulated by centrifugal feedback from higher brain areas (Linster and Cleland, 2016; Padmanabhan et al., 2016). The complexity of M/T neural circuits suggests that these neurons not only participate in odor representation, but may also carry information related to learning, odor values, behavioral context, and task demands (Gire et al., 2013b; Nunez-Parra et al., 2014). Recent studies focusing on the function of M/Ts at the single-cell level in awake behaving mice demonstrated that activity in single $\mathrm{M} / \mathrm{Ts}$ (1) carries information about both odor identity and odor value (Li et al., 2015, 2017), (2) displays transient/long-term plasticity during learning (Abraham et al., 2014; Gschwend et al., 2015; Chu et al., 2016), and (3) is highly dependent on the brain state and context (Yamada et al., 2017; Jordan et al., 2018; Koldaeva et al., 2019).

The piriform cortex, which is further divided into the anterior piriform cortex (APC) and the posterior piriform cortex (PPC), is a higher olfactory center that receives direct input from the OB. The piriform cortex encodes information about the identity, intensity, and timing of odors (Bolding and Franks, 2017, 2018; Jiang et al., 2017). More importantly, the piriform cortex plays a major role in odor preference learning, odor pattern separation, olfactory learning, odor fear memory, and the processing of odor objects (Wilson and Sullivan, 2011; Bao et al., 2016; Schaffer et al., 2018; Meissner-Bernard et al., 2019).

However, it remains unknown whether odor representation in the population of $\mathrm{OB} \mathrm{M} / \mathrm{Ts}$ or piriform pyramidal neurons varies with the task demands. For example, some tasks merely require detection of odors but other tasks require identification or discrimination of odors. Here, we investigate this issue by using fiber photometry to record the population calcium signals from $\mathrm{M} / \mathrm{Ts}$ and piriform pyramidal neurons in awake behaving mice under different task demands. Our results demonstrate that odor representation in the M/T population is dependent on task demands; this finding is supported by electrophysiological recordings of spikes and local field potentials (LFPs). Strikingly, a task-demand-dependent neural representation is also found in the PPC but not the APC, indicating different functional specificity in these two important higher olfactory centers.

\section{Materials and Methods}

\section{Animals}

Male C57BL/6J and Thy1-cre [FVB/N-Tg(Thyl-cre)1V1n/J] mice aged $8-16$ weeks old were used in this study. The number of mice used in each experiment is reported in the Results. All the mice were bred in the animal facilities of Xuzhou Medical University and housed in a vivarium with a $12 \mathrm{~h} \mathrm{light/dark} \mathrm{cycle,} \mathrm{with} \mathrm{lights} \mathrm{on} \mathrm{at} \mathrm{8:00} \mathrm{A.M.} \mathrm{After} \mathrm{surgery,}$ mice were housed individually for at least 1 week before further experiments to allow for recovery. Experiments were performed during the light cycle. Food and water were available ad libitum except during the periods when the mice were performing behavioral tasks, when water was instead received in the experimental chamber; however, if the mice did not obtain $2 \mathrm{ml}$ of water during a behavioral session, additional water was provided in a dish in the cage. All mice used for the behavioral study were weighed daily and received sufficient water during the behavioral sessions to maintain $>80 \%$ of their pre-water-restriction weight. All experimental procedures were performed in accordance with protocols submitted to and approved by the Xuzhou Medical University Institutional Animal Care and Use Committee.

\section{Surgery for implantation of tetrodes and optical fibers}

Mice were briefly anesthetized with pentobarbital (i.p., $0.09 \mathrm{mg} / \mathrm{g}$ bodyweight). Depth of anesthesia was verified by toe pinch. The mouse was mounted in a stereotaxic frame and the fur on the surface of the scalp from the midline of the orbits to the midpoint between the ears was removed. A hole was drilled above the right $\mathrm{OB}$, APC, or PPC for the implant (OB, AP: $4.0 \mathrm{~mm}$; ML: $1.0 \mathrm{~mm}$; APC, AP: $2.1 \mathrm{~mm}$; ML: $2.0 \mathrm{~mm}$; PPC, AP: $-2.0 \mathrm{~mm}$; ML: $4.0 \mathrm{~mm}$ ).

For spike and LFP recordings, tetrodes were implanted into the OB. Tetrodes were lowered to an average depth of $1.8-2.0 \mathrm{~mm}$, targeting the ventral mitral cell layer (Li et al., 2015, 2017). Each tetrode consisted of four polyimide-coated nichrome wires (single-wire diameter, item \#PF000591. RO-800, 0.0005 inch, $12.7 \mu \mathrm{m}$, coating 1/4 hard PAC, Sandvik) connected to a 16-channel electrode interface board (EIB-16, NeuraLynx). A screw inserted $1 \mathrm{~mm}$ posterior from bregma and $1 \mathrm{~mm}$ lateral to the midline served as the reference electrode and was connected to the ground. Recordings were made during tetrodes implantation to ensure optimal placement within the ventral mitral cell layer. The signals recorded from the tetrodes were sent to a headstage and amplified by a 16-channel amplifier (Plexon DigiAmp; bandpass: 1-5000 Hz, 2000X gain), and then sampled at $40 \mathrm{kHz}$ by a Plexon OmniPlex recording system. Finally, the tetrodes were sealed to the bone with dental acrylic. A custom-made aluminum headplate was attached to the skull with stainless steel screws and dental cement.

\section{Virus injection and fiber implant}

In Thy1-cre mice, the mitral cell layer of the $\mathrm{OB}$ was injected with AAVDIO-GCaMP6s (BrainVTA). In C57BL/6J mice, layer 2/3 of the APC/ PPC was injected with AAV-CaMKII $\alpha$-GCaMP6s (BrainVTA). All injections were made with a glass pipette and the injecting volume and velocity were controlled by a microsyringe pump (Stoelting Quintessential Injector). Virus solutions ( $300 \mathrm{nl}$ ) were injected slowly $(50 \mathrm{nl} / \mathrm{min})$ into the mitral cell layer of the OB (AP: $4.0 \mathrm{~mm}$; lateral: $1.0 \mathrm{~mm}$; DV: $0.8-1.1 \mathrm{~mm}$ ) or layer $2 / 3$ of the APC/PPC (APC, AP: $2.1 \mathrm{~mm}$; lateral: 2.0 $\mathrm{mm}$; DV: $4.0 \mathrm{~mm}$; PPC, AP: $-2.0 \mathrm{~mm}$; lateral: $4.0 \mathrm{~mm}$; DV: $4.6 \mathrm{~mm}$ ). The glass pipette was left in place for an additional $10 \mathrm{~min}$ before being slowly withdrawn. After the virus injection, an optical fiber [outer diameter (o.d.) $200 \mu \mathrm{m}$; numerical aperture (NA) 0.37; NEWDOON] was implanted in the mitral cell layer of the OB or layer $2 / 3$ of the APC/PPC at the same coordinates. The optical fiber was fixed in place with dental acrylic and an aluminum head plate was attached to the skull for headfixed recordings. Mice were housed individually for at least $10 \mathrm{~d}$ after surgery for recovery and to allow time for the expression of GCaMP6s.

\section{Spike and LFP recordings}

Recordings were started after the mice had recovered from surgery for at least $10 \mathrm{~d}$. Awake mice were head-fixed with two horizontal bars (fixed to the headplate by 2 screws) and were able to maneuver on an airsupported free-floating Styrofoam ball (Thinkerbiotech). For spike recordings, the signals from the tetrodes were sent to a headstage and amplified by a 16-channel amplifier (Plexon DigiAmp; bandpass filtered at $300-5000 \mathrm{~Hz}, 2000 \times$ gain), and sampled at $40 \mathrm{kHz}$ by a Plexon OmniPlex recording system. For LFP recordings, the signals were amplified $(2000 \times$ gain; Plexon DigiAmp), filtered at $0.1-300 \mathrm{~Hz}$, and sampled at 
$1 \mathrm{kHz}$. Odor stimulation event markers were recorded alongside the spikes/LFP via the Plexon OmniPlex recording system.

\section{Fiber photometry}

Fluorescence emissions were recorded with a fiber photometry system (Thinkerbiotech) using methods similar to those described in previous studies (Guo et al., 2015; Li et al., 2016; Zhou et al., 2017; Zhang et al., 2019). Briefly, a laser beam from a $488 \mathrm{~nm}$ laser (OBIS 488LS, Coherent) was reflected by a dichroic mirror (MD498, Thorlabs), focused through an objective lens (10×, NA: 0.3; Olympus), and then coupled to an optical commutator (Doric Lenses). An optical fiber (200 mm o.d., NA: $0.37,1.5 \mathrm{~m}$ long) coupled the light between the commutator and the implanted optical fiber. The laser power was adjusted to be $40-60 \mu \mathrm{W}$ at the tip of the optical fiber. GCaMP6s fluorescence emissions were bandpass filtered (MF525-39, Thorlabs) and detected by a photomultiplier tube (R3896, Hamamatsu). An amplifier (C7319, Hamamatsu) was used to convert the photomultiplier tube current output to voltage, which was further filtered through a low-pass filter ( $35 \mathrm{~Hz}$ cutoff; Brownlee, 440). The analog voltage signals were digitized at $500 \mathrm{~Hz}$ and recorded by fiber photometry software (Thinkerbiotech).

\section{Odor application}

Odors were presented by an odor delivery system (Thinkerbiotech). Four odor pairs were used: isoamyl acetate versus 2-heptanone, phenyl acetate versus benzaldehyde, dimethylbutyric acid versus $n$-heptane acid and n-pentanol versus 2-pentanone (Sinopharm Chemical Reagent). All odorants were dissolved in mineral oil at $1 \% \mathrm{v} / \mathrm{v}$ dilution. In the odordelivery mode, a stream of charcoal-filtered air flowed over the oil in the odor-delivery system, and was then diluted to $1 / 20$ by an olfactometer. Odor presentation was synchronously controlled by the data acquisition system via a solenoid valve driven by a digital-to-analog converter. Air or odorized air was delivered to the nose at a constant rate of $11 / \mathrm{min}$ to eliminate the effect of airflow. For each odor, $10-15$ trials were presented with an intertrial interval of $30 \mathrm{~s}$. The duration of each odor presentation was $2 \mathrm{~s}$.

\section{Overview of training and behavioral tasks}

After animals recovered from surgery, mice underwent a series of behavioral tasks, similar to those described in our previous study (Sun et al., 2019). Water was removed from the mouse's cage and behavioral training began once a mouse reached $80-85 \%$ of its pre-water-deprivation bodyweight. The mice were head-fixed with two horizontal bars (fixed with headplate by 2 screws) and were able to maneuver on an airsupported free-floating Styrofoam ball. At the beginning of training, water-deprived mice were required to lick a water delivery tube, and obtained water reinforcement if they licked at any time during the odor stimulation. Before training on the go/no-go task, mice were trained to perform a go/go task. In this go/go session, mice would receive a water reward if they licked within the odor stimulation period $(2 \mathrm{~s})$ when either of the two odors in the pair was present. Then the mice were trained to perform the go/no-go task in which they were required to discriminate between the two odors to receive the water reward (see Fig. $5 B$; Li et al., 2015, 2017). The mice learned to lick a tube when a reinforced odor (S+) was present and to not lick the tube when the unreinforced odor $(\mathrm{S}-$ ) was present. Thus, if an $\mathrm{S}+$ was presented and the mice responded with licking (Hit), a water reward was delivered through the lickport; if they did not lick in response to the S+ (Miss) the water reward was not delivered. If an $\mathrm{S}-$ was presented, water was never delivered, regardless of the mouse's actions [if they licked, false alarm (FA); if they did not lick, correct rejection (CR); see Fig. 5B]. Hit and CR are classed as correct responses, whereas Miss and FA are classed as incorrect responses. The animal's performance was evaluated in blocks of 20 trials, with 10 trials per odor and $\mathrm{S}+$ and $\mathrm{S}-$ presented randomly within each block. The percentage correct value for each block represents the percentage of trials in which the odors were correctly discriminated and associated with the appropriate behavioral action. Each session included 6-10 blocks of 20 trials. Once the mice had learned to discriminate between $\mathrm{S}+$ and $\mathrm{S}-$, they were ready for the reversed go/no-go task in which $S+$ and $S-$ were switched. Electrophysiological signals or calcium signals were simultaneously recorded during all tasks.

\section{Histology}

For verification of viral expression, frozen brain sections were prepared. Mice were anesthetized with pentobarbital (i.p., $80 \mathrm{mg} / \mathrm{kg}$ bodyweight) and transcardially perfused with $20 \mathrm{ml}$ of $0.9 \%$ saline, followed by $20 \mathrm{ml}$ of $4 \%$ paraformaldehyde (PFA) in PBS $(0.1 \mathrm{M}, \mathrm{pH} 7.4)$. After perfusion, brains were harvested and postfixed for $24 \mathrm{~h}$ in PFA at $4^{\circ} \mathrm{C}$ and then were cryoprotected with $30 \%$ sucrose in PBS until the tissue sank. Brain tissue was then embedded in OCT compound and sectioned to $30 \mu \mathrm{m}$ thick on an upright Leica cryostat. Tissue sections were mounted on slides and imaged by confocal scanning microscopy (Zeiss, LSM710).

\section{Experimental design and statistical analysis}

Behavioral performance. For the go/no-go task, performance was calculated for each training day (1 session). Performance across all trials (see Figs. $5 C, 7 A$ ), was calculated as follows: (number of Hit trials + number of CR trials)/total number of trials, including all Hit, Miss, CR, and FA trials.

Off-line spike sorting and statistics of the unit data. Spikes were sorted from the raw data with Offline Sorter V4 software (Plexon). The separation of different units was performed by principal component analysis (see Fig. 3). A unit was classified as a single unit if $<0.75 \%$ of the interspike intervals were $<1 \mathrm{~ms}$ (Jeanne et al., 2013; Li et al., 2017). This results in unimodal firing rate distributions. The data $2 \mathrm{~s}$ before and $4 \mathrm{~s}$ after the onset of each odor stimulation event were extracted, and a peristimulus time histogram (PSTH) was generated by averaging the spike firing rate within $100 \mathrm{~ms}$ bins (see Fig. $3 E, F$ ). The spontaneous firing rate was calculated by averaging across the spikes fired during the $2 \mathrm{~s}$ before odor stimulation (baseline); the odor-evoked firing rate was calculated by averaging across the spikes fired during the $2 \mathrm{~s}$ after the onset of odor stimulation. To test whether an odor evoked a significant response, we used a paired $t$ test to compare the baseline firing rate with the odor-evoked firing rate across all the trials for each cell-odor pair (10 trials for each odor and thus 10 samples for each group). If the $P$ value was $>0.05$, the particular cell-odor pair was defined as nonresponsive. If the $P$ value was $<0.05$, the cell-odor pair was defined as responsive and was further categorized as excitatory (if the odor-evoked firing rate was greater than the baseline firing rate; see Fig. $3 E$ ) or inhibitory (if the odor-evoked firing rate was lower than the baseline firing rate; see Fig. $3 F)$.

Analysis of LFP signals. A MATLAB script was used to analyze the LFP signals. As in previous studies (Li et al., 2015; Zhou et al., 2017), LFP signals were divided into different frequency bands: theta $(2-12 \mathrm{~Hz})$, beta (15-35 Hz), and gamma (low gamma, 36-65 Hz; high gamma, 66-95 $\mathrm{Hz}$ ). In this study, we focused on the beta and high gamma bands for further analysis since odors usually evoke strong and reliable responses within these two frequency bands (see Fig. $4 C-F$ ). To assess the odorevoked beta band and high gamma band LFP responses, we selected a window starting $2 \mathrm{~s}$ before the onset of the odor presentation and ending $4 \mathrm{~s}$ after the onset of the odor stimulation presentation (see Fig. 4C-F). To obtain high resolution in both the time and frequency domains, this time course was divided into segments of $1 \mathrm{~s}$ duration with $90 \%$ overlap. Time-frequency transformation was performed on the $1 \mathrm{~s}$ windows. The spectral power from all frequencies included within the bandwidth was averaged. For each trial, the baseline was normalized to 1 , and all the trials for each odor stimulation were averaged based on the normalized data (Fig. 4E,F).

Analysis of photometry data. Data were exported as MATLAB.mat files for further analysis. The data were segmented based on the onset of odor stimulation within individual trials. We derived the values of fluorescence change $(\Delta F / F)$ by calculating $\left(F-F_{0}\right) / F_{0}$, where $F_{0}$ is the baseline fluorescence signal averaged over a 5 -s-long control time window, which preceded the onset of odor stimulation. $\Delta F / F$ values are presented as heat maps or trial-averaged plots (Fig. $1 C, D$ ).

ROC analysis. Receiver operating characteristic (ROC) analysis was used to assess the classification of the responses evoked by odor pairs. ROCs were estimated using the roc function from the MATLAB exchange. The area under the ROC (auROC) is a nonparametric measure of the discriminability of two distributions. We used auROC to assess the classification of the two odors within an odor pair. The area under the 

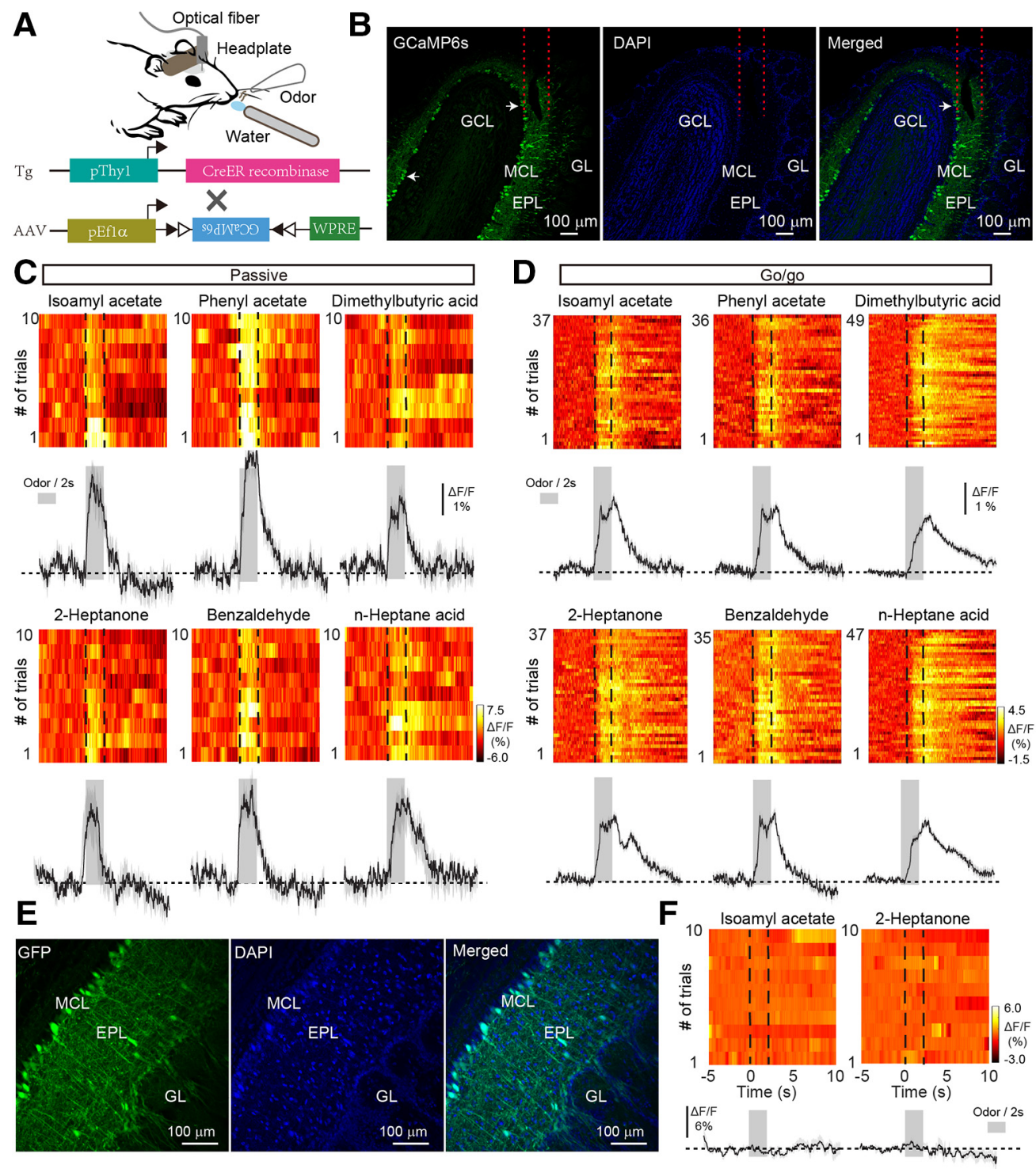

Figure 1. Odor response profiles in the $M / T$ population of the $O B$ under passive exposure and go/go task conditions. $A$, Schema of the experimental paradigm. To record the odor-evoked calcium responses of mitral cells, AAV-expressing GCaMP6s was injected into the olfactory bulb of Thy1-Cre mice. B, Expression of GCaMP6s in the mitral cells. Within the 0B, GCaMP6s is expressed in the mitral cells (left). DAPI stain highlights the cell layers of the $\mathrm{OB}$ (middle). The merged image (right) shows colocalization of GCaMP6s (green) and DAPI (blue). Arrows indicate two examples of M/Ts expressing GCaMP6s. GL, Glomerular layer; EPL, external plexiform layer; MCL, mitral cell layer. GCL, granule cell layer. C, D, Trial-to-trial pseudocolored heat maps (top) and trial-averaged traces (bottom; mean \pm SEM) of the calcium responses evoked by different odors with passive exposure $(\boldsymbol{C})$ and in the go/go task $(\boldsymbol{D})$. Dashed lines in heat maps and gray boxes in trial-averaged traces represent the 2 s odor presentation period. $\boldsymbol{E}$, Expression of EGFP in mitral cells. AAV-expressing EGFP was injected into the OB of Thy1-Cre mice. $\boldsymbol{F}$, Heat maps (top) and trial-averaged traces (bottom) from a representative animal-odor pair during passive exposure in a Thy1-EGFP mouse.

ROC curve was defined as from 0.5 to 1.0 . A value of 0.5 indicates completely overlapping distributions, whereas a value of 1 indicates perfect discriminability.

Calculation of differences in $\Delta \mathrm{F} / \mathrm{F} /$ firing rate/power. We used difference in $\Delta F / F /$ firing rate/power to assess the extent of the divergence in the responses to the two odors within an odor pair. The responses evoked by the two odors were defined as Res 1 and Res2, respectively. The difference in $\Delta F / F /$ firing rate/power was calculated as follows: ABS (Res1 - Res2)/ [ABS (Res1) + ABS (Res2)], where ABS represents the absolute value; Res 1 and Res2 represent the responses evoked by odor 1 and odor2, respectively. Based on the calculation defined above, a difference of 1 usually indicates that one odor evoked an excitatory response, and the other odor evoked an inhibitory response. An extreme situation that also results in a difference of 1 is when one odor evokes a response and the other odor evokes zero response. However, this situation did not occur in our dataset. The data, including $\Delta F / F$, spike firing rate and spectral power of the LFP, were averaged over trials before calculating the difference in
$\Delta F / F /$ firing rate/power. All data in present study are presented as the mean \pm SEM.

\section{Results}

Convergence of odor-evoked responses of the M/T population in a go/go task

To record the population activity of $\mathrm{M} / \mathrm{Ts}$, calcium signals were monitored with the genetically encoded $\mathrm{Ca}^{2+}$ indicator GCaMP6s in awake head-fixed mice using fiber photometry. A previous study reported that, in the $\mathrm{OB}$, the Thyl promoter is specifically expressed in M/Ts (Arenkiel et al., 2007). We therefore used a Thy1-Cre line injected with an adeno-associated virus (AAVDIO-GCaMP6s; Fig. 1A). Consistent with our previous study (Zhang et al., 2019), 3 weeks after viral injection there was extensive expression of GCaMP6s in the mitral cell layer of the OB (Fig. $1 B)$. Thus, using this method, we can selectively record odor- 

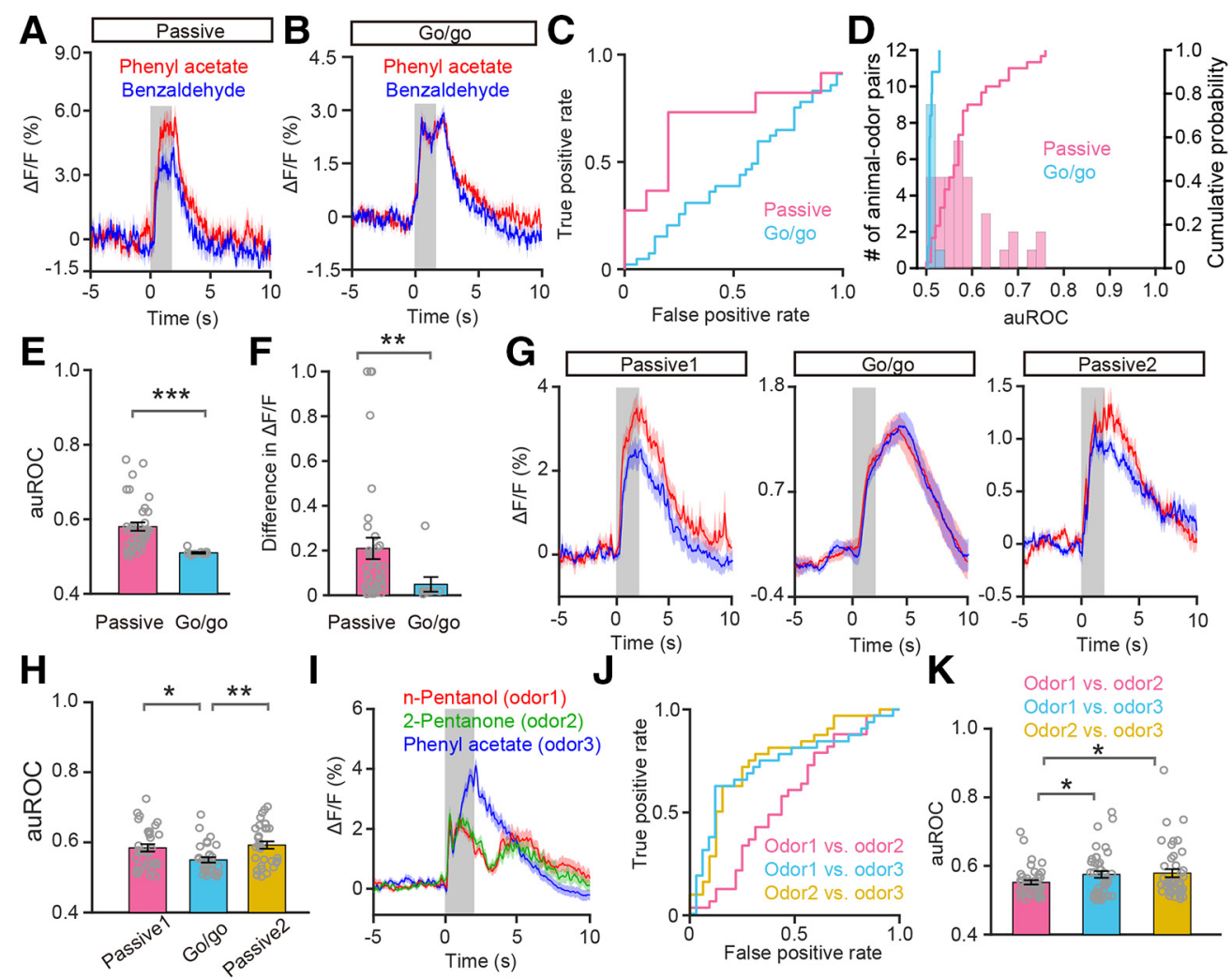

Figure 2. Odor responses in the M/T population converge in the go/go task. $\boldsymbol{A}, \boldsymbol{B}, 0$ dor response traces evoked by one odor pair (phenyl acetate vs benzaldehyde) with passive exposure ( $\boldsymbol{A}$ ) and in the go/go task conditions $(\boldsymbol{B})$. Odor presentation is indicated by the gray box. $\boldsymbol{C}$, The ROC graph for $\Delta F / F$ shown in $\boldsymbol{A}$ and $\boldsymbol{B}$. The data were estimated from the averaged $\Delta F / F$ during the $2 \mathrm{~s}$ odor delivery period. $\boldsymbol{D}$, Histograms and cumulative probability of the auROCs for all the animal-odor pairs in the passive exposure $(n=36$ animal- odor pairs from 9 mice) and the go/go task ( $n=10$ animal-odor pairs from 5 mice) conditions. $\boldsymbol{E}, \boldsymbol{F}$, Comparison of auROCs $(\boldsymbol{E}) /$ difference in $\Delta F / F(\boldsymbol{F})$ between passive exposure and the go/go task conditions across all animal- 0 dor pairs. Each gray circle represents the auROC value/difference in $\Delta F / F$ for a single animal- odor pair. $\boldsymbol{E}$, Mann-Whitney test, ${ }^{* * *} p<0.0001(z=-4.164) ;(\boldsymbol{F})$ Mann-Whitney test, ${ }^{* *} p<0.01(z=-2.554)$. $\mathbf{G}, 0$ dor response traces from a representative mouse evoked by one odor pair with passive exposure (Passive1), in the go/go task, and with passive exposure after the go/go task (Passive2).

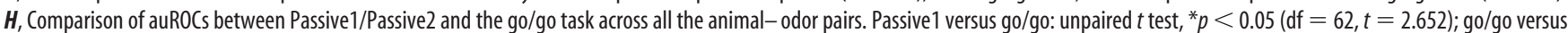
Passive2: unpaired $t$ test, ${ }^{* *} p<0.01(\mathrm{df}=62, t=-3.279)$. II, Odor response traces evoked by the odor pair (n-pentanol vs 2-pentanone, odors delivered during the go/go task) and a third, untrained odor (phenyl acetate). $J$, The ROC graph for $\Delta F / F$ shown in $I . K$, Comparison of auROCs across all the animal-odor pairs. Odor 1 and odor2 represent all the odor pairs delivered during the go/go task and odor3 represents all the untrained odors. Odor1/odor2 versus odor1/odor3: paired $t$ test, ${ }^{*} p<0.05$ ( $\mathrm{df}=41, t=-2.235$ ); odor1/odor2 versus odor2/odor3: paired $t$ test, ${ }^{*} p<0.05$ (df $=41, t=-0.427)$.

evoked responses from the $\mathrm{M} / \mathrm{T}$ population located beneath the optical fiber.

Studies using in vivo electrophysiological recordings or twophoton calcium imaging in anesthetized or awake mice have shown that some M/Ts display increased activity in response to odor stimulation whereas other $\mathrm{M} / \mathrm{T}$ s display decreased activity at the single cell level (Rinberg et al., 2006; Kato et al., 2012; Kikuta et al., 2013; Wachowiak et al., 2013; Economo et al., 2016; Li et al., 2017; Yamada et al., 2017; Jordan et al., 2018). However, no previous study has reported the odor-evoked population response from $\mathrm{M} / \mathrm{T}$ s using fiber photometry. We first characterized the population response of M/Ts to odors in awake head-fixed mice without a task demand (passive exposure). Although excitatory responses were observed for most of the odors delivered (Fig. 1C), inhibitory responses were also observed. Among the nine mice tested, seven mice responded to all eight odors (4 odor pairs) exclusively with excitation, one mouse responded exclusively with inhibition, and one mouse responded with excitation or inhibition depending on the odor presented. Therefore, odors could evoke either excitatory or inhibitory population responses in $\mathrm{M} / \mathrm{Ts}$, consistent with previous findings using other techniques (Rinberg et al., 2006; Bolding and Franks, 2017; Li et al., 2017; Yamada et al., 2017; Jordan et al., 2018). Mice that ex- pressed only enhanced green fluorescent protein (EGFP) did not show any changes in fluorescence after odor stimulation (Fig. $1 E, F)$, indicating that the signals observed in mice expressing GCaMP6s were not motion artifacts.

Next, we used fiber photometry to record the odor-evoked responses of the $\mathrm{M} / \mathrm{T}$ population when mice were engaged in a go/go task. In this task, mice were trained to lick the waterdelivery tube after odor presentation, and both odors in an odor pair were associated with water reinforcement. Figure $1 D$ shows representative traces from one mouse induced by the same three odor pairs presented in Figure 1C. A total of 10 animal-odor pairs (from 5 mice) were recorded and all of the responses were excitatory; no inhibitory responses were observed. That is, only simple odor-evoked excitatory responses were observed during the go/go task.

Interestingly, unlike the responses during the passive exposure, where different odors within a pair evoked somewhat different responses, the responses evoked by different odors within a pair during the go/go task appeared similar. An example is shown in Figure 2, $A$ and $B$. To compare the classification of the responses evoked by odor pairs with passive exposure and in the go/go task, we performed the ROC analysis (Fawcett, 2006). For the example shown in Figure 2, $A$ and $B$, the auROC, representing 
the difference in responses to the odor pair, was smaller in the go/go condition than in the passive exposure condition (Fig. 2C). ROC analysis of all animal-odor pairs showed that the difference between the auROC values for passive exposure (pink) and the go/go task (cyan) was statistically significant ( $p<0.0001$, MannWhitney test, $n=36$ animal-odor pairs from 9 mice in the passive exposure, $n=10$ animal-odor pairs from 5 mice in the go/go task; Fig. $2 D, E)$. To quantify the extent of the response difference evoked by odors within a pair, we compared the difference in $\Delta F / F$, calculated as the difference in averaged $\Delta F / F$ during the $2 \mathrm{~s}$ odor stimulation. We found that the difference in $\Delta F / F$ was smaller during the go/go task than with passive exposure $(p=$ 0.009 , Mann-Whitney test, $n=36$ animal-odor pairs from 9 mice in the passive exposure condition, $n=10$ animal-odor pairs from 5 mice in the go/go task; Fig. $2 F$ ). Therefore, these data indicate that the odor-evoked $\mathrm{M} / \mathrm{T}$ population responses elicited by two different odors tend to converge during the go/go task.

Because we performed the passive exposure experiment first, and the go/go task second, this poses the possibility that the observed changes in response to odors within a pair simply reflects habituation. To exclude this possibility, we performed an additional experiment in which we first presented the odors to mice under passive exposure condition (Fig. 2G, Passive1), then in the go/go task (Fig. 2G, Go/go), and finally under passive exposure condition again (Fig. 2G, Passive2). Same odorants were used in the three states (passive-go/go-passive). We obtained data from the same mice in all three states. The results clearly show that odor classification in the go/go state was significantly worse than in either of the two passive states (Passive1 vs go/go: $p=0.01$; go/go vs Passive2: $p=0.002$; unpaired $t$ tests, $n=64$ animalodor pairs from 8 mice; Fig. $2 H$ ), indicating that the observed difference between responses to odor pairs in the passive state and the go/go task reflects a different representational strategy and not simply habituation.

The convergent response in the go/go task might be because the animals know that the specific odor pairs in the odor pair are associated with the lick-reward. However, it might also be because the animals have been trained to generalize that the presence of any odorant signifies a lick-reward. To clarify this, we performed an experiment in which a third, untrained odor (Fig. $2 I$, odor3) was presented during the go/go task. We found that the response difference between the first odor pair (Fig. 2I, odor 1 and odor2) in the go/go task was significantly smaller than the difference between either odor1 or odor2 and odor3 (evaluated by auROC; odor 1/odor 2 vs odor 1/odor $3: p=0.031$; odor $1 /$ odor 2 vs odor2/odor3: $p=0.017$; paired $t$ tests, $n=42$ animal-odor pairs from 7 mice; Fig. $2 J, K)$. These results indicate that the poor classification performance for odor pairs during the go/go task is because animals know that the odors in a specific odor pair are associated with a lick-reward, not because they have generalized their learning to lick in response to any odorant.

\section{Spikes recorded from M/Ts display convergent odor-evoked responses in the go/go task}

Changes in GCaMP6s fluorescence measured by fiber photometry reflect the neural activity of the $\mathrm{M} / \mathrm{T}$ population beneath the fiber. To assay the responses of single M/Ts, we performed extracellular recordings (Fig. 3A). Tetrodes were placed into the mitral cell layer and single $\mathrm{M} / \mathrm{T}$ units were isolated as described previously (Li et al., 2014, 2015, 2017). Figure 3B shows examples of two single $\mathrm{M} / \mathrm{Ts}$ sorted from tetrode recordings. We observed both excitatory and inhibitory responses to odor presentation with passive exposure and in the go/go task (Fig. 3C,D). Figure $3 E$ displays the raster plots and corresponding PSTHs depicting the different responses evoked in a single unit by passive exposure to two different odors (phenyl acetate vs benzaldehyde). Figure $3 F$ shows that the same odor pair evoked similar responses during the go/go task. The ROC curves assessing the difference in firing rate for these two examples are shown in Figure 3G. AuROC values were smaller during the go/go task than with passive exposure $(p<0.0001$, Mann-Whitney test, $n=73$ cell-odor pairs from 5 mice in the passive exposure condition, $n=133$ cell-odor pairs from 10 mice in the go/go task; Fig. $3 H, I$ ). Moreover, we found a significant difference in firing rate between the passive exposure condition and the go/go task $(p<0.0001$, MannWhitney test, $n=73$ cell-odor pairs from 5 mice in the passive exposure condition, $n=133$ cell-odor pairs from 10 mice in the go/go task; Fig. 3J). Therefore, consistent with the results obtained via fiber photometry, single units recorded from the mitral cell layer also display convergent responses during the go/go task.

\section{Odor-evoked beta but not high gamma oscillations display convergent responses in the go/go task}

Whereas neural activity from single cells provides information important for sensory representation and encoding, neuronal oscillations arise from local circuit processing and provide a syntactical framework for communication between brain areas (Buzsáki et al., 2012; Pesaran et al., 2018). LFP oscillations in the $\mathrm{OB}$, especially beta $(15-35 \mathrm{~Hz})$ and high gamma oscillations (65-95 Hz), reflect circuit processing for odor detection and discrimination in awake rodents performing specific odor-related tasks (Martin and Ravel, 2014; Kay, 2015; Frederick et al., 2016). We asked whether the convergent responses of $\mathrm{M} / \mathrm{Ts}$ to odors during the go/go task are reflected in odorant responses measured as changes in the power of LFP oscillations. Figure 4, $A$ and $B$, shows example LFP traces (raw, filtered beta, and high gamma oscillations) recorded from one mouse during passive exposure (Fig. 4A) and in the go/go task (Fig. 4B). During odor presentation, the power of beta oscillations increased but the power of high gamma oscillations decreased (Fig. 4C-F). Interestingly, during passive exposure, the beta LFP responses to the two odors differed but the high gamma responses were similar (Fig. 4E). However, both beta and high gamma responses to the two odors were similar during the go/go task (Fig. $4 F$ ). Next, we used the auROC to quantify the differences in LFP power to the two odorants during passive exposure and in the go/go task (Fig. 4G,H). For odor-evoked beta oscillations, the auROC was significantly smaller in the go/go task than during passive exposure condition, but for high gamma oscillations there was no difference between the two conditions (beta: $p=0.002$, high gamma: $p=0.540$, Mann-Whitney test, $n=20$ animal-odor pairs from 5 mice in the passive exposure condition, $n=21$ animal-odor pairs from 10 mice in the go/go task; Fig. 4I). When we examined the difference in power evoked by the two odors within a pair, we found that this difference was similar in the passive exposure and go/go conditions for both the beta and the high gamma band (beta: $p=0.481$, high gamma: $p=0.389$, MannWhitney test, $n=20$ animal-odor pairs from 5 mice during the passive exposure condition, $n=21$ animal-odor pairs from 10 mice in the go/go task; Fig. $4 J)$. Together, our data indicate that convergent LFP power responses to odor pairs are found during the go/go task for beta oscillations, but not high gamma oscillations. 


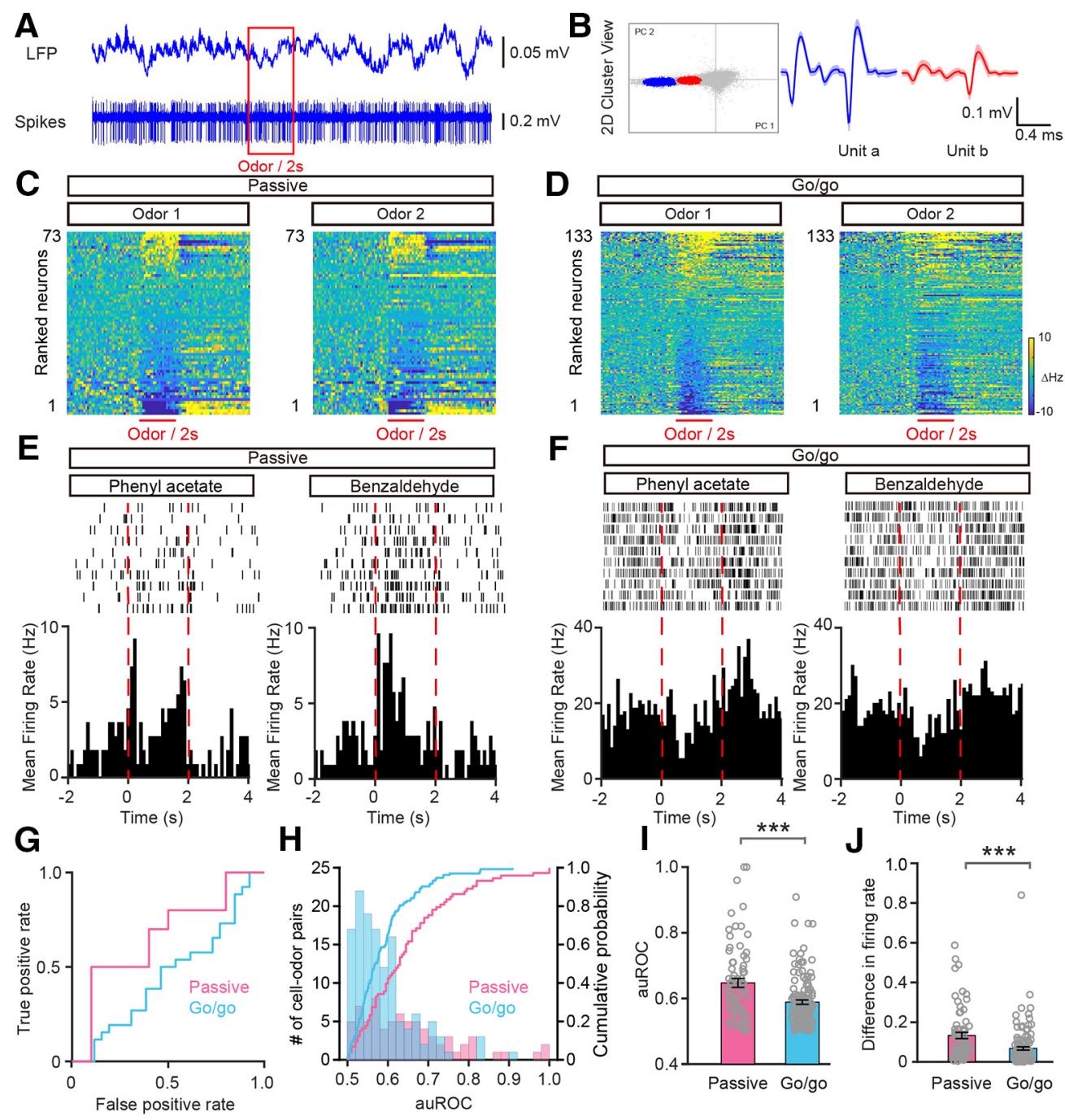

Figure 3. Convergent odor-evoked firing in M/Ts in the go/go task. $A$, Example of raw traces of $L F P$ and spikes from one odor stimulation trial. $\boldsymbol{B}$, Example of spike sorting using PCA scan clustering of the extracellular voltage recorded by tetrodes, resulting in separation of two units (unit a, blue; unit b, red). $\boldsymbol{C}, \boldsymbol{D}$, Heat maps of firing rates averaged across all trials and further averaged across odorants for each neuron, ranked by mean odor-evoked firing rate, for odor 1 in both the passive exposure $(\boldsymbol{C} ; n=73)$ and go/go task $(\boldsymbol{D} ; n=133)$ datasets. Odor1 and odor 2 represent the two odors from the odor pairs. $\boldsymbol{E}, \boldsymbol{F}$, Example of firing induced by one odor pair (phenyl acetate vs benzaldehyde) during passive exposure $(\boldsymbol{E})$ and in the go/go task $(\boldsymbol{F})$. Top, raster plot. Bottom, PSTHs for the firing rate. The odor stimulation ( $2 \mathrm{~s}$ ) is indicated by dashed red lines. $\boldsymbol{G}$, The ROC graph for the M/T firing rates shown in $\boldsymbol{A}$ and $\boldsymbol{B}$. $\boldsymbol{H}$, Histogram and cumulative probability of the auROCs for all the cell- odor pairs under the passive exposure and the go/go task conditions. $I, J$, Comparison of auROCs $(I) /$ difference in firing rate $(J)$ between the passive exposure condition $(n=73$ cell- 0 dor pairs from 5 mice) and the go/go task ( $n=133$ cell- odor pairs from 10 mice). $I$, Mann-Whitney test, ${ }^{* * *} p<0.0001(z=-3.775) ;(J)$ Mann-Whitney test, ${ }^{* * *} p<0.0001(z=-4.439)$.

Improved differentiation of the $\mathrm{M} / \mathrm{T}$ population responses to rewarded and unrewarded odors during an odor discrimination task

Why does the M/T population show convergent responses to odor pairs during the go/go task? We hypothesized that divergence of the M/T population response to odors within a pair depends on the task demands. During the go/go task, the two presented odors are rewarded equally. Therefore, they represent the same outcome and no discrimination of the odors is necessary. If this hypothesis stands, odors associated with different reward outcomes should evoke different responses. To test this hypothesis, we performed a go/no-go task experiment (see Materials and Methods). We analyzed the responses of the M/T population using fiber photometry of GCaMP6s fluorescence while head-restrained mice learned to respond differently to odors in a go/no-go odor discrimination task (Fig. 5A). For the go/no-go task, thirsty mice were trained to lick the water tube for water during $S+$ delivery. When exposed to $S-$, mice refrained from licking because of the absence of water reward during this period. To temporally separate the response to the odor from the reward period, the $2 \mathrm{~s}$ odor delivery was followed by a $2 \mathrm{~s}$ waiting period during which water was not delivered (Fig. 5B). Task performance was assessed by calculating the percentage of correct responses to the $\mathrm{S}+$ and $\mathrm{S}-$ odors in blocks of 20 trials in which 10 $\mathrm{S}+$ and $10 \mathrm{~S}-$ odors were delivered randomly. The performance of mice improved from near chance levels (50\% correct) in Block 1 to well above the learning threshold ( $80 \%$ correct) in Block 4 (Fig. $5 C$ ). Figure $5 D$ shows the odor-evoked responses of the M/T population for a representative mouse learning to discriminate the odors in a pair ( $\mathrm{S}+$ : phenyl acetate; $\mathrm{S}-$ : benzaldehyde). The traces were sorted into trials where the mouse was learning to differentiate the odorants (left, blocks with an average of $60 \%$ correct responses, learning state) and trials where mice were proficient in discriminating the odorants (right, blocks with an average of $85 \%$ correct responses, proficient state). The two odors evoked similar responses before the mouse learned to discrimi- 

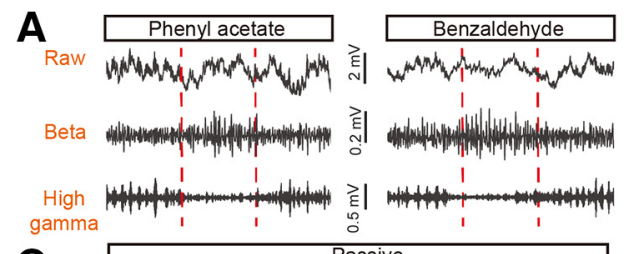

B
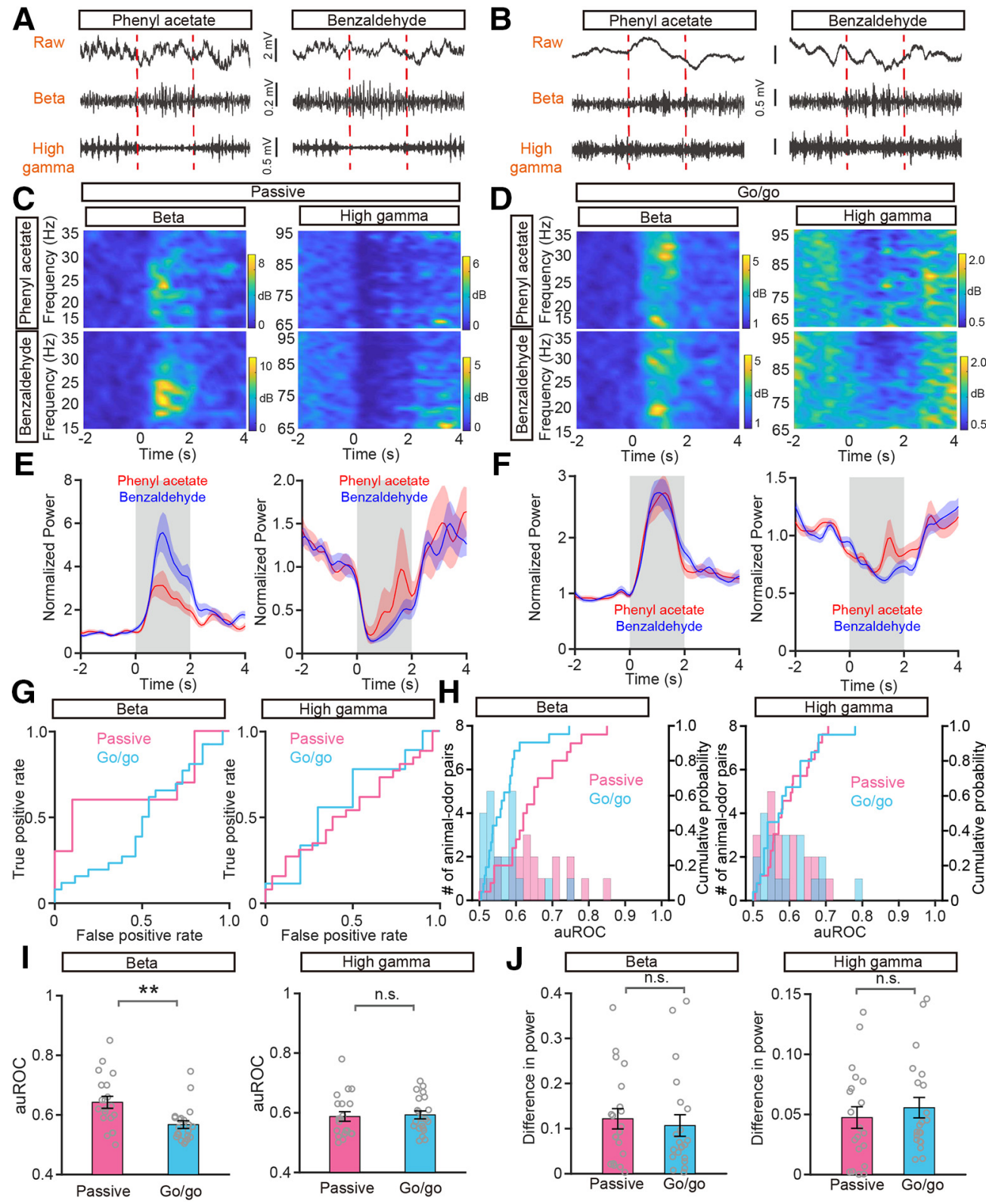

Figure 4. Odor-evoked beta and high gamma responses under passive exposure and go/go task conditions. $\boldsymbol{A}, \boldsymbol{B}$, Raw traces (top) and filtered beta (middle) and high gamma (bottom) oscillations in the LFP signals in a representative mouse in respond to presentation of a pair of odors (phenyl acetate vs benzaldehyde) during passive exposure $(\boldsymbol{A})$ and in the go/go task (B). $\boldsymbol{C}, \boldsymbol{D},($ hanges in the power spectrum elicited by the two odors under passive exposure $(\boldsymbol{C})$ and go/go task (D) conditions for beta (top) and high gamma (bottom) oscillations. $\boldsymbol{E}$, $\boldsymbol{F}$, Averaged (across 10 trials) normalized traces for the beta (left) and high gamma (right) responses during passive exposure $(\boldsymbol{E})$ and in the go/go task $(\boldsymbol{F})$. Red and blue indicate the responses evoked by phenyl acetate and benzaldehyde, respectively. $\boldsymbol{G}$, The ROC graphs for the beta (left) and high gamma (right) responses shown in $\boldsymbol{E}$ and $\boldsymbol{F}$. $\boldsymbol{H}$, Histograms and cumulative probability of the auROCs for beta (left) and high gamma (right) responses under passive exposure and go/go task conditions. $\boldsymbol{I}, \boldsymbol{J}$, Comparison of auROCs $(\boldsymbol{I}) /$ difference in power $(\boldsymbol{J})$ for beta (left)/high gamma (right) responses under passive exposure $(n=20$ animal-odor pairs from 5 mice $)$ and in the go/go task ( $n=21$ animal- odor pairs from 10 mice). $I$, Mann-Whitney test, ${ }^{* *} p<0.01(z=-3.118)$ and n.s. $(z=-0.613) ;(J)$ Mann-Whitney test, n.s. $(z=-0.704)$ and n.s. $(z=-0.861)$.

nate them; however, the responses to the $\mathrm{S}-$ were smaller once the mouse had learned to discriminate the odors. Figure $5 D$ shows the individual traces and Figure $5 E$ shows the average changes in fluorescence. Analysis of the odorant-induced changes in GCaMP6s fluorescence showed that the auROC values were larger after the mouse had learned to discriminate the odors (Fig. $5 F$ ).

The development of a divergent response to the odorants as the animal learned was observed consistently for other animalodor pairs. The average response traces for the learning and proficient states for all animal-odor pairs are shown in Figure $5 G$. Both the auROC values and the difference in $\Delta \mathrm{F} / \mathrm{F}$ across all the mouse-odor pairs were significantly larger in the proficient state than in the learning state in the go/no-go task (auROC: $p=0.005$, difference in $\Delta F / F: p=0.005$, Wilcoxon's sign rank test; $n=10$ animal-odor pairs from 5 mice; Fig. $5 H-J)$. These data demonstrate that a divergent response to odorants in the $\mathrm{M} / \mathrm{T}$ population depends on the task demands, specifically, whether the mouse needs to discriminate the odors to obtain a reward. These observations are consistent with previous studies reporting that, at the singlecell level, M/Ts display improved pattern separation after active sensory learning (Doucette and Restrepo, 2008; Doucette et al., 2011; Gschwend et al., 2015).

In the very early stages of the go/no-go task (i.e., in the first block), the animals likely do not realize they are performing a new 
A
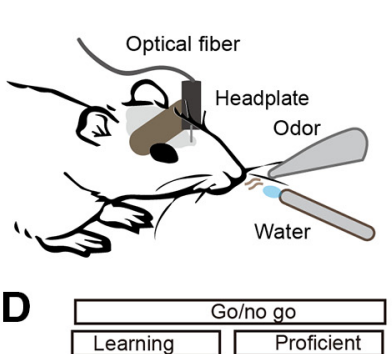
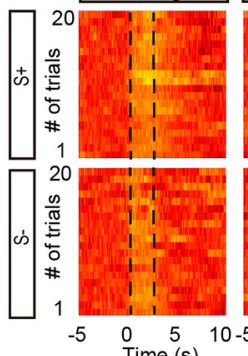

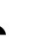

G

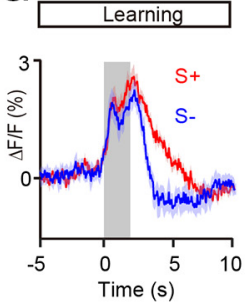

J

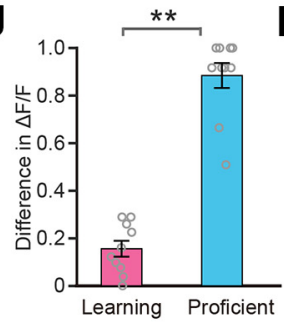

B

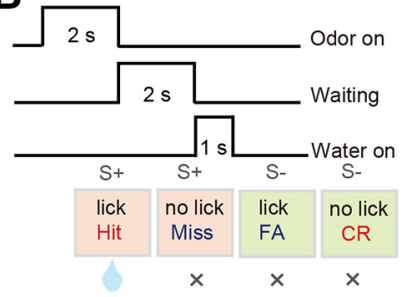

E
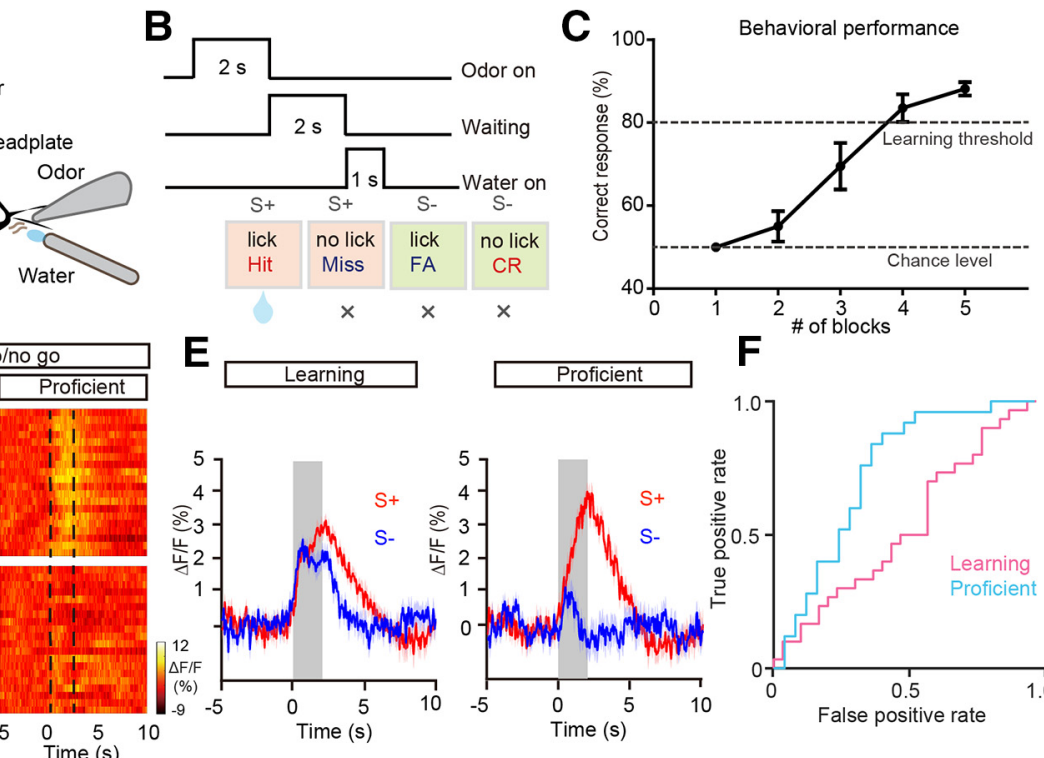

$F$

\section{$F$}

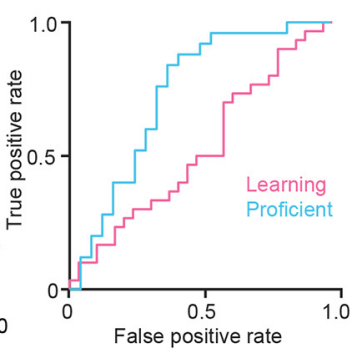

H
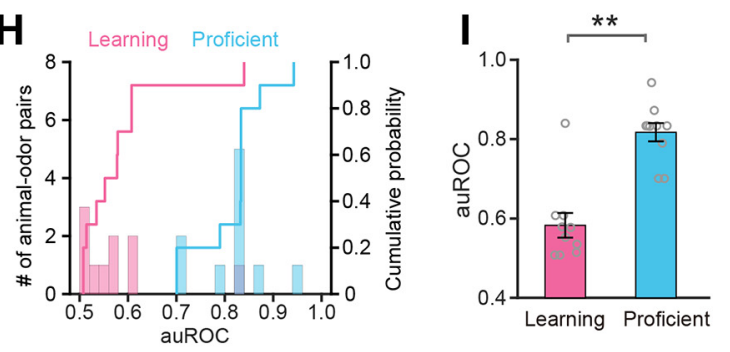

K
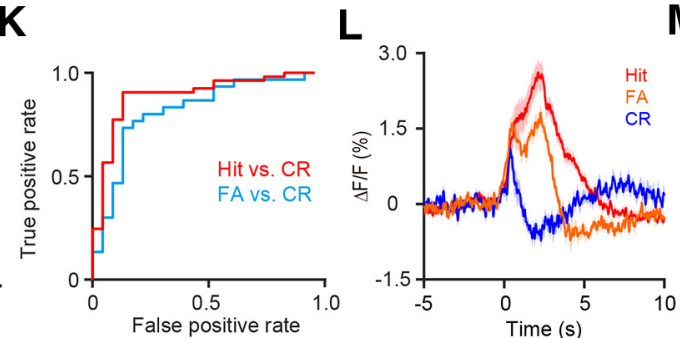

M

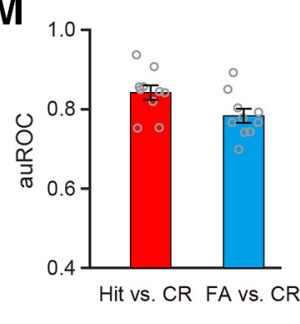

Figure 5. Odor responses in the $M / T$ population show improved differentiation after the mice learn to discriminate the odors in a pair in the go/no-go task. $A$, Diagram of the experimental paradigm. $B$, Schematic of the go/no-go task sequence. Odor was applied for $2 \mathrm{~s}$, followed by $2 \mathrm{~s}$ waiting time, followed by delivery of the water reward. $C$, The odor discrimination performance during the go/no-go task across all the sessions recorded ( $n=8$ animal-odor pairs from 4 mice). The mean percentage correct responses across sessions are plotted for each block ( 20 trials). The chance level and learning threshold are indicated by dashed lines. $\boldsymbol{D}-\boldsymbol{F}$, Heat maps $(\boldsymbol{D})$, trial-averaged traces $(\boldsymbol{E})$, and the ROC graph $(\boldsymbol{F})$ for odor responses induced by $\boldsymbol{S}+$ and $\boldsymbol{S}-$ in the learning state and in the proficient state during the go/no-go task, from a representative mouse. $G$, Averaged traces of odor responses across all animal-odor pairs ( $n=10$ animal-odor pairs from 5 mice). $\boldsymbol{H}$, Histograms and cumulative probability of auROCs in the learning state and in the proficient state during the go/no-go task across all animal- odor pairs. $I, J$, Comparison of auROCs (I)/difference in $\Delta F / F(J)$ between the learning state and the proficient state during the go/no-go task across all animal-odor pairs. $I$, Wilcoxon's sign rank test, ${ }^{* *} p<0.01(z=-2.807) ;(J)$ Wilcoxon's sign rank test, ${ }^{* *} p<0.01(z=-2.803) . K$, The ROC graph of odor responses induced during Hit and CR trials, and FA and CR trials from a representative mouse. $L$, Averaged traces of odor responses of Hit, $F A$, and CR trials across all animal-odor pairs. $M$, The auROCs for Hit versus CR trials and FA versus CR trials.

task since their performance is around chance levels (Fig. $5 C$ ). Then, their performance begins to increase, indicating that the mice have realized that they are performing a new task and are in the process of learning. Thus, to confirm the different odor representations during the learning and proficient states in the go/ no-go task, we reanalyzed the data excluding the first block. Consistent with the results shown in Figure 5, $I$ and $J$, both the auROC and the difference in $\Delta F / F$ were significantly different in the learning and proficient states (auROC: $0.61 \pm 0.03$ and $0.82 \pm 0.02$ for the learning and proficient states, respectively; $\mathrm{df}=19, t=2.82, p=0.005$, paired $t$ test. Difference in $\Delta F / F$ : $0.16 \pm 0.03$ and $0.88 \pm 0.05$ for the learning and proficient states, respectively; $\mathrm{df}=19, t=2.81, p=0.005$, paired $t$ test).

In the go/no-go task, the behavioral responses on most of the trials were Hit, CR, or FA, with very few Miss trials (4/1041,
$0.38 \%)$. Using a method similar to that described in a previous study (Ramirez-Gordillo et al., 2018), we calculated the auROC for Hit/CR trials and for FA/CR trials: an example is shown in Figure $5 \mathrm{~K}$. The averaged odor-evoked response traces are shown in Figure $5 \mathrm{~L}$. We found that the auROC was significantly different from 0.5 (the diagonal) for both Hit/CR and FA/CR (Hit/CR: $\mathrm{df}=$ 9, $t=18.036, p<0.0001$; FA/CR: $\mathrm{df}=9, t=16.276, p<0.0001$, one-sample $t$ test, $n=10$ animal-odor pairs from 5 mice; Fig. $5 M$ ). Thus, the odor-evoked calcium response performs relatively well at classifying correct from incorrect responses, indicating that the response reflects odor value as opposed to odor identity. This finding is consistent with the results from a previous study in which the LFP power was evaluated (Ramirez-Gordillo et al., 2018).

During the go/no-go task, the learning state usually occurred during the early trials in a session while the proficient state oc- 

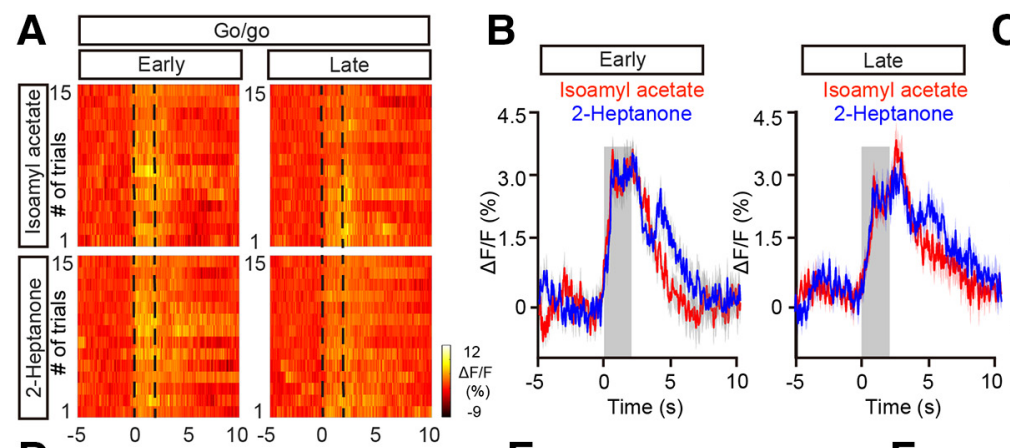

C
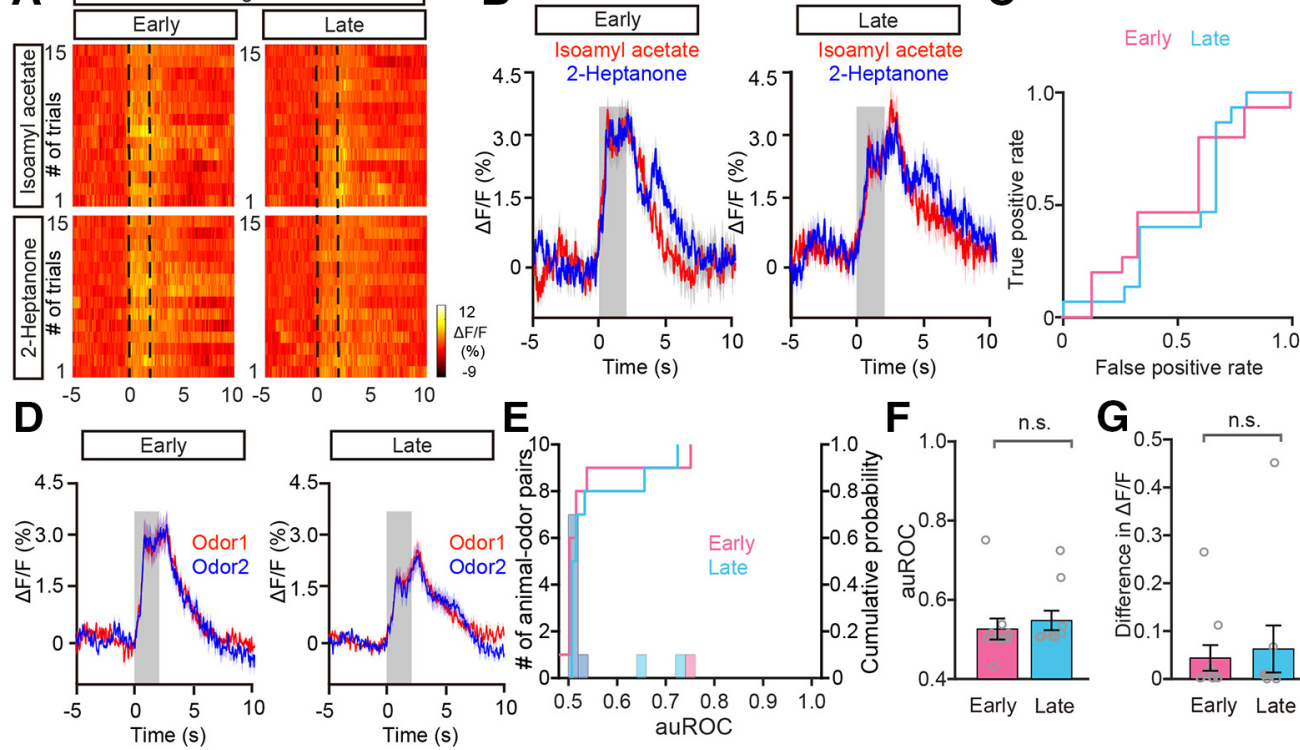

F

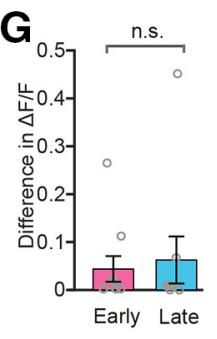

Figure 6. Changes in odor-evoked responses during the go/go task. $\boldsymbol{A}-\boldsymbol{C}$, Heat maps $(\boldsymbol{A})$, trial-averaged traces $(\boldsymbol{B})$ and ROC graph $(\boldsymbol{C})$ of responses evoked by the two odors in the early trials and in the late trials during the go/go task, from a representative mouse. $\boldsymbol{D}$, Averaged odor response traces across all animal-odor pairs ( $n=10$ animal-odor pairs from 5 mice). $\boldsymbol{E}$, Histograms and cumulative probability of auROCs in early trials and late trials during the go/go task across all animal- odor pairs. $F, G$, Comparison of auROCs $(\boldsymbol{F}) /$ difference in $\Delta F / F(G)$ between early trials and late trials in the go/go task across all animal-odor pairs. $F$, Wilcoxon's sign rank test, n.s. $(z=-0.905)$; (G) Wilcoxon's sign rank test, n.s. $(z=-0.663)$.

curred later in the session (Fig. 5C). This raises the question of whether the difference in divergent odorant responses between these behavioral states is due to general behavioral state differences such as thirst. To address this question, we analyzed the data focusing on odor responses during early trials (the first 30 trials) and late trials (the last 30 trials) obtained from the go/go task where animals also receive water and become satiated (Fig. $6 A$ ). The odor-evoked responses were similar between the two odors during both the early trials and the late trials in a representative mouse (Fig. 6B-E). Further analysis indicated that neither the auROC values nor the difference in $\Delta \mathrm{F} / \mathrm{F}$ across all the animal-odor pairs were significantly different between early trials and late trials during the go/go task (auROC: $p=0.366$, difference in $\Delta F / F: p=0.508$, Wilcoxon's sign rank test; $n=10$ animal-odor pairs from 5 mice; Fig. $6 F, G$ ). Therefore, the taskdemand-dependent neural representation in the $\mathrm{M} / \mathrm{T}$ population is established by learning-related plasticity as opposed to behavioral states, such as thirst, that differ between the beginning and end of the session.

\section{Divergence of the responses to odors within a pair develops} again after the rewarded and unrewarded odors are reversed Another prediction of the task-demand-dependent representation strategy is that the odor responses should be associated with odor value but not odor identity. To test this prediction, we recorded calcium signals from mice engaged in the odor discrimination task when the rewarded and unrewarded odors were switched. All the mice learned to discriminate the switched odors successfully (Fig. $7 A$ ), Figure $7 B$ shows the M/T population responses from a representative mouse responding to the new $\mathrm{S}+$ (previously $\mathrm{S}-$ ) and new $\mathrm{S}-$ (previously $\mathrm{S}+$ ). Similar to the results from the forward go/no-go task, the responses evoked by the two odors within a pair were similar in the learning state, and differentiated in the proficient state (Fig. $7 C-F$ ). Across all the animal-odor pairs, both the auROC values and the difference in $\Delta F / F$ were significantly larger in the proficient state than in the learning state in the reversed go/no-go task (auROC: $p=0.005$, difference in $\Delta F / F: p=0.005$, Wilcoxon's sign rank test; $n=10$ animal-odor pairs from 5 mice; Fig. $7 G, H)$. Importantly, the divergent responses to the two odors in the proficient state resulted from a reduced response to the $S-$ (previously the $S+$ ). In summary, our data suggest that, during both forward and reversed go/no-go tasks, the M/T cell population displays divergent responses to odors with different reward associations after learning. This is consistent with studies showing that the synchronized firing of mitral cells carries information about odor value but not odor identity (Doucette et al., 2011; Nunez-Parra et al., 2014).

Task-demand-dependent neural representation is routed into PPC but not APC

Because both the APC and the PPC are higher olfactory centers that receive dense direct inputs from M/Ts (Igarashi et al., 2012), we next asked whether the pyramidal neurons in the APC and the PPC display the same divergence in response to odorants under different task demands. To address this question, we first measured the population calcium signal from APC pyramidal neurons by injecting AAV-CaMKII $\alpha$-GCaMP6s virus into the APC of $\mathrm{C} 57 \mathrm{BL} / 6 \mathrm{~J}$ mice and recording fluorescence changes using fiber photometry (Fig. 8A). Consistent with our previous study (Zhou et al., 2017), pyramidal cells in the APC expressed GCaMP6s (Fig. 8B).

During the passive exposure condition, although most of the responses to the two odorants were similar (Fig. $8 C-F$ ), we found a small number of differential responses to the two odorants (Fig. $8 G$; 2 of 14 animal-odor pairs had auROC values larger than 0.70 ). This is partially consistent with previous studies indicating that the firing rate of single APC neurons can carry information on odor identity (Miura et al., 2012; Gire et al., 2013a; Bolding and Franks, 2017; Jiang et al., 2017). During the go/go task, the responses to the two odorants were similar. Overall, neither the auROCs nor the difference in $\Delta F / F$ were significantly different between the two conditions (auROC: $p=0.667$, difference in 

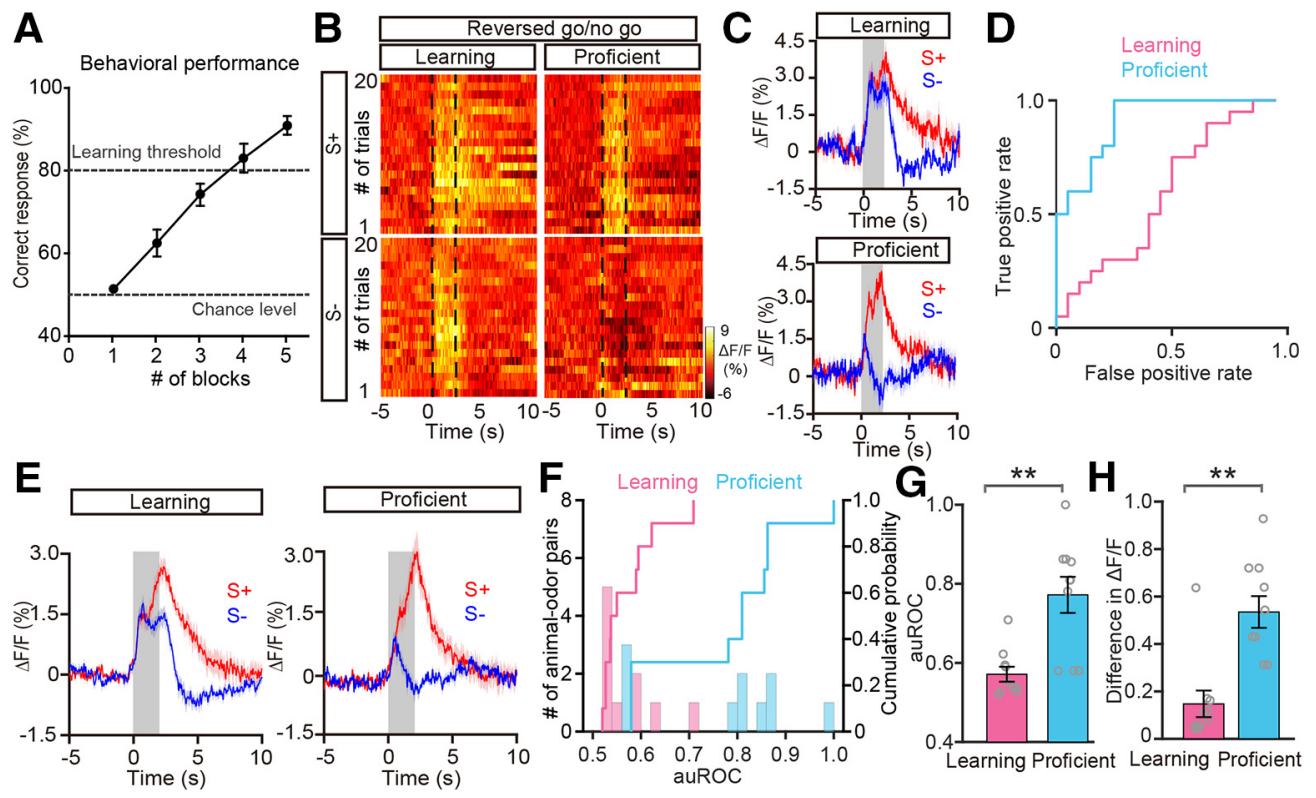

Figure 7. Improved differentiation is also observed in the reversed go/no-go task. $A$, The odor discrimination performance during the reversed go/no-go task across all the sessions recorded $(n=$ 8 animal-odor pairs from 4 mice). $\boldsymbol{B}-\boldsymbol{D}$, Heat maps $(\boldsymbol{B})$, trial-averaged traces $(\boldsymbol{C})$, and the ROC graph $(\boldsymbol{D})$ of odor responses induced by $\boldsymbol{S}+$ and $S-$ in the learning state and in the proficient state during the reversed go/no-go task from a representative mouse. $\boldsymbol{E}$, Averaged traces of odor responses across all animal-odor pairs ( $n=10$ animal-odor pairs from 5 mice). $\boldsymbol{F}$, Histograms and cumulative probability of auROCs in the learning state and the proficient state during the reversed go/no-go task across all animal- odor pairs. $\boldsymbol{G}, \boldsymbol{H}, \mathrm{Comparison}$ of auROCs $(\boldsymbol{G}) /$ difference in $\Delta F / F$ $(\boldsymbol{H})$ between the learning state and the proficient state during the reversed go/no-go task across all animal-odor pairs. G, Wilcoxon's sign rank test, ${ }^{* *} p<0.01(z=-2.805) ;(\boldsymbol{H})$ Wilcoxon's sign rank test, ${ }^{* *} p<0.01(z=-2.803)$.
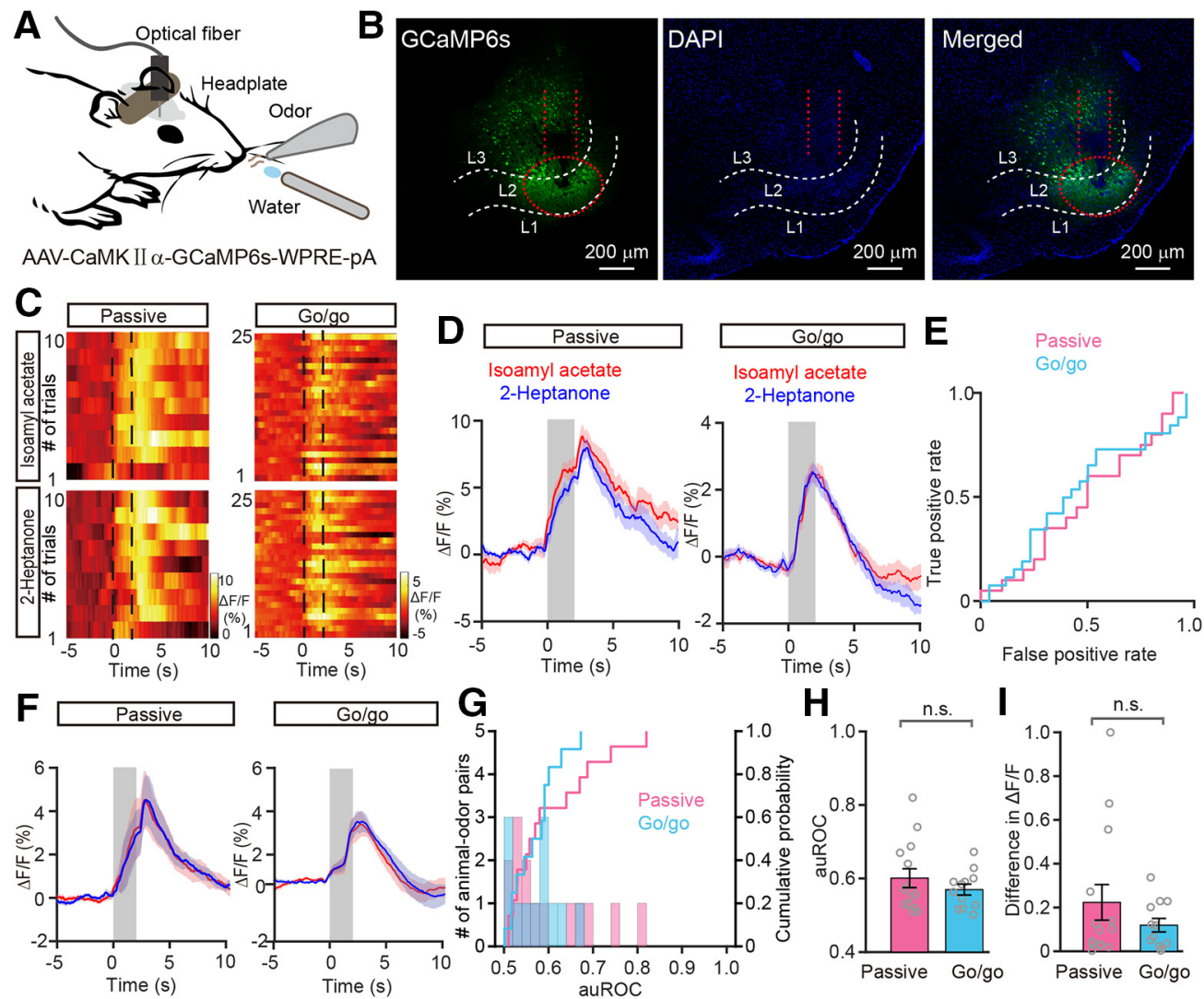

Figure 8. Changes in the odor responses of APC pyramidal neurons under passive exposure and go/go task conditions. $A$, Schema of the experimental paradigm. To record the odor-evoked calcium response of APC pyramidal neurons, AAV-expressing CaMKIl $\alpha$-GCaMP6s was injected into the APC of C57 BL/6J mice. $\boldsymbol{B}$, Expression of GCaMP6s in APC pyramidal neurons. $\boldsymbol{C}-\boldsymbol{E}$, Heat maps $(\boldsymbol{C}$, trial-averaged traces $(\boldsymbol{D})$, and ROC graph $(\boldsymbol{E})$ of responses evoked by the two odors under passive exposure and go/go task conditions. $\boldsymbol{F}$, Averaged traces of odor responses across all animal- 0 dor pairs. G, Histograms and cumulative probability of auROCs under passive exposure $(n=14$ animal-odor pairs from 9 mice) and go/go task conditions across all animal- 0 dor pairs $(n=12$ animal- odor pairs from 8 mice). $\boldsymbol{H}, \boldsymbol{I}$, Comparison of auROCs $(\boldsymbol{H}) /$ difference in $\Delta F / F(\boldsymbol{I})$ between the passive exposure and the go/go task conditions across all animal- odor pairs. $\boldsymbol{H}$, Mann-Whitney test, n.s. $(t=-0.463) ;(I)$ Mann-Whitney test, n.s. $(z=-0.360)$. 

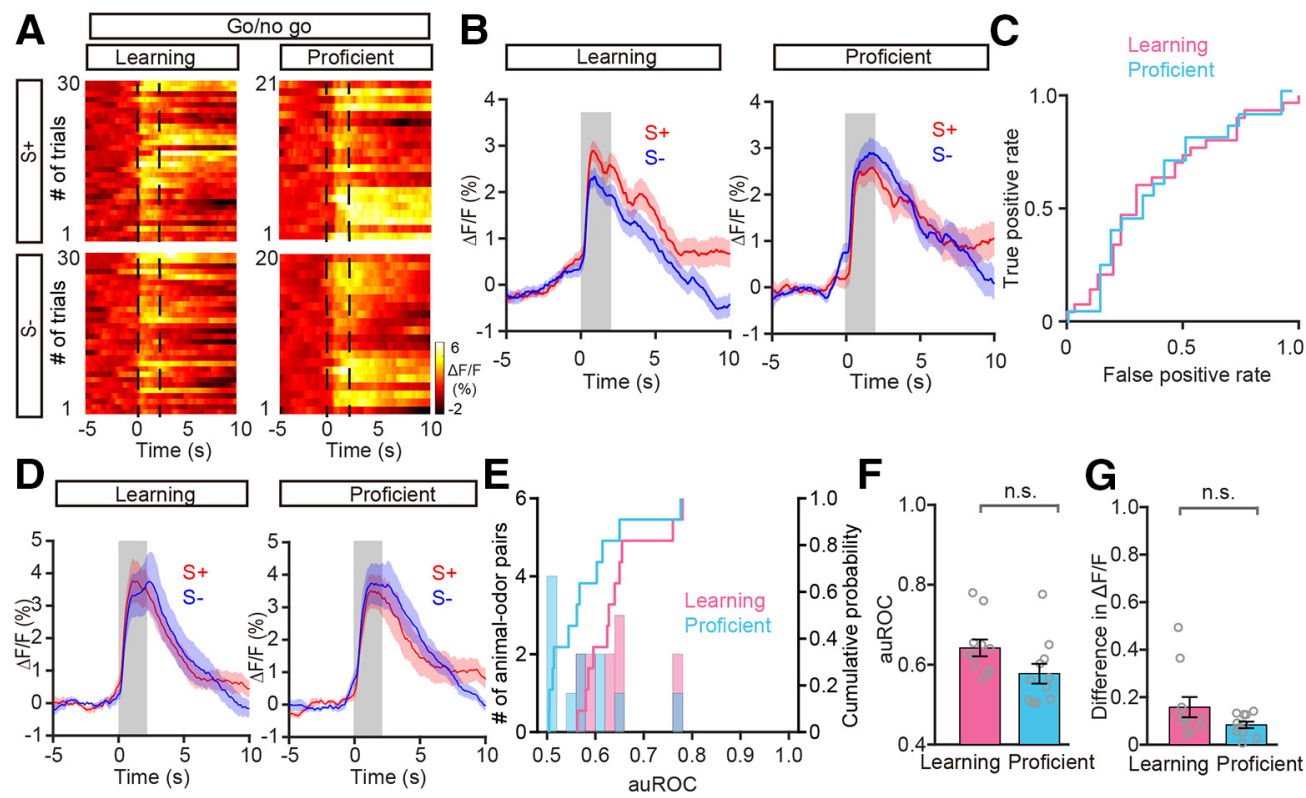

Figure 9. Absence of differentiation in the APC pyramidal neurons during the go/no-go task. $\boldsymbol{A}-\boldsymbol{C}$, Heat maps $(\boldsymbol{A})$, trial-averaged traces $(\boldsymbol{B})$, and the ROC graph $(\boldsymbol{C})$ for responses induced by $S+$ and $S-$ in the learning state and in the proficient state during the go/no-go task, from a representative mouse. $\boldsymbol{D}$, Averaged traces of odor responses across all animal-odor pairs $(n=11$ animal-odor pairs from 8 mice). $\boldsymbol{E}$, Histograms and cumulative probability of auROCs in the learning state and in the proficient state during the go/no-go task across all animal- odor pairs. $\boldsymbol{F}, \boldsymbol{G}$, Comparison of auROCs $(\boldsymbol{F}) /$ difference in $\Delta F / F(G)$ between the learning state and the proficient state in the go/no-go task across all animal- odor pairs. $\boldsymbol{F}$, Paired $t$ test, n.s. (df $=10, t=1.763)$; (G) Wilcoxon's sign rank test, n.s. $(z=-1.156)$.

$\Delta F / F: p=0.742$, Mann-Whitney test, $n=14$ animal-odor pairs from 9 mice in the passive exposure condition, $n=12$ animalodor pairs from 8 mice in the go/go task; Fig. $8 H, I)$. During the go/no-go task, we did not observe development of differential responses to the two odorants as the mice learned to differentiate the odorants (Fig. 9A-E). Neither the auROCs nor the differences in $\Delta F / F$ were significantly different between the learning state and the proficient state (auROC: $p=0.108$, paired $t$ test, difference in $\Delta F / F: p=0.248$, Wilcoxon's sign rank test; $n=11$ animal-odor pairs from 8 mice; Fig. $9 F, G$ ). Therefore, these data suggest that, unlike M/Ts, APC pyramidal neurons do not show task-demand-dependent changes in the neural representation of odor information.

Finally, we tested whether pyramidal neurons in the PPC have task-demand-dependent neural representations. As in the APC, we recorded the calcium signals from PPC pyramidal neurons with fiber photometry by injecting AAV-CaMKII $\alpha$-GCaMP6s virus into the PPC of C57BL/6J mice (Fig. 10A). Figure $10 B$ shows neurons in the PPC expressing GCaMP6s. As in the APC, although most of the responses to the two odorants were similar (Fig. 10C-F), some differential responses to the two odorants were also found during passive exposure (Fig. 10G; 5 of 30 animal-odor pairs had auROC values $>0.70$ ). However, neither the auROCs nor the differences in $\Delta F / F$ were significantly different between passive exposure condition and the go/go task (auROC: $p=0.899$, unpaired $t$ test, difference in $\Delta F / F: p=0.774$, MannWhitney test; $n=30$ animal-odor pairs from 10 mice in the passive exposure condition, $n=20$ animal-odor pairs from 7 mice in the go/go task; Fig. $10 \mathrm{H}, \mathrm{I})$. During the go/no-go task, we found development of response divergence: responses to the two odors were similar in the learning state but diverged in the proficient state (Fig. 11A-E). Interestingly, whereas differentiation appeared soon after the start of odor delivery in the OB (Fig. 5G), differentiation appeared relatively late in the PPC, after odor removal (Fig. $11 B, D$ ). Further statistical analysis showed that, al- though there were no significant differences between the learning and proficient states during odor exposure (the period from 0 to $2 \mathrm{~s}$ after odor stimulation, auROC: $p=0.121$, paired $t$ test, difference in $\Delta F / F: p=0.08 ; n=15$ animal-odor pairs from 7 mice; Fig. $11 F, G)$, both the auROCs and the difference in $\Delta F / F$ were significantly larger in the proficient state compared with the learning state during the period $4-6 \mathrm{~s}$ after odor stimulation (auROC: $p=0.010$, paired $t$ test, difference in $\Delta F / F: p=0.020$, Wilcoxon's sign rank test; $n=15$ animal-odor pairs from 7 mice; Fig. $11 H-K)$. Furthermore, when odorant valence in the go/ no-go task was reversed, divergent responses to the odorants developed in the same $4-6 \mathrm{~s}$ period (Fig. 12A-E). After mice learned to discriminate the odor pair, the auROCs increased and the difference in $\Delta F / F$ increased significantly (auROC: $p=0.169$, paired $t$ test, difference in $\Delta F / F: p=0.028$, paired $t$ test; $n=9$ animal-odor pairs from 4 mice; Fig. $12 F, G$ ). Thus, the PPC pyramidal neuron population also contains task-dependent neural representations and carries information on odor value. These data indicate that the neural representation in the PPC changes depending on the task demands.

\section{Discussion}

The neural activity of $\mathrm{M} / \mathrm{T}$ s shows robust plasticity during learning, and odor representation in M/Ts is highly dynamic under different brain states and behavioral contexts (Doucette and Restrepo, 2008; Fontanini and Katz, 2008; Doucette et al., 2011; Li et al., 2011, 2017; Kato et al., 2012; Chu et al., 2016; Jordan et al., 2018). Here, we used fiber photometry to demonstrate that odor representation in the $\mathrm{M} / \mathrm{T}$ population is highly dependent on task demands. During passive exposure, in the absence of task demands, calcium signals from the $\mathrm{M} / \mathrm{T}$ population show divergent responses to the two odors within a pair. These divergent responses vanish during the go/go task, in which mice are rewarded for licking regardless of the identity of the odorant. However, robust divergent responses develop again as the animal learns to 
A

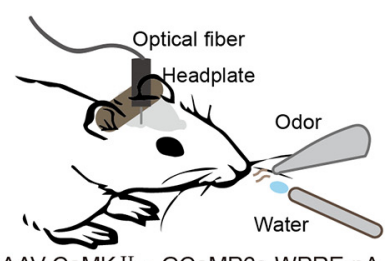

B

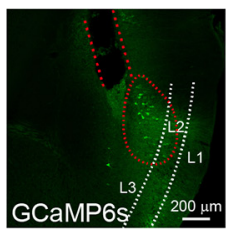

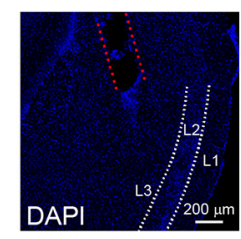

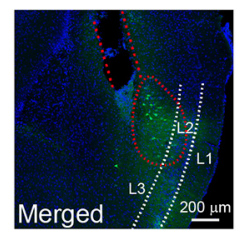

AAV-CaMK II $\alpha$-GCaMP6s-WPRE-pA
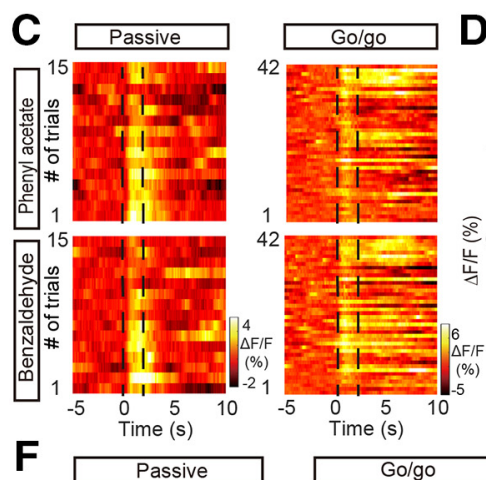

D
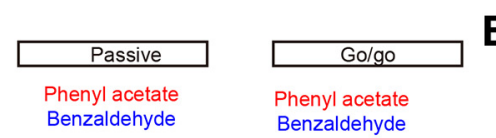

$\mathbf{E}$
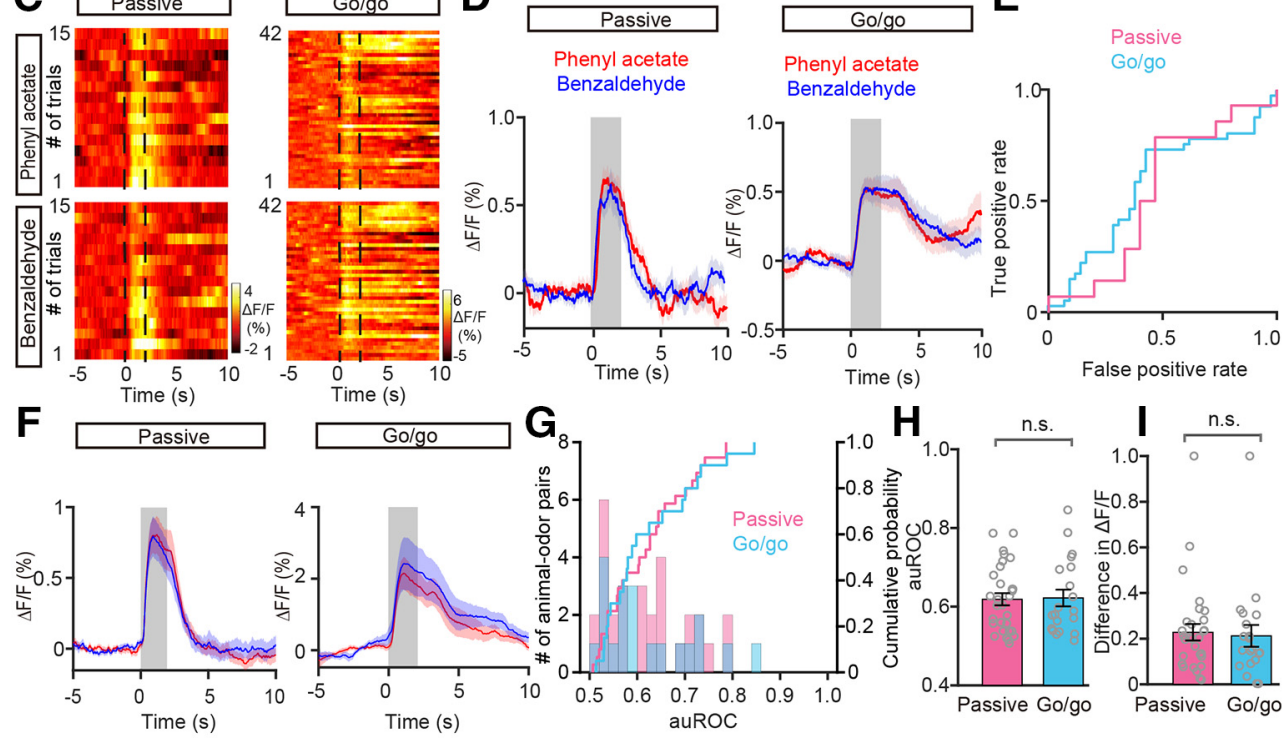

H

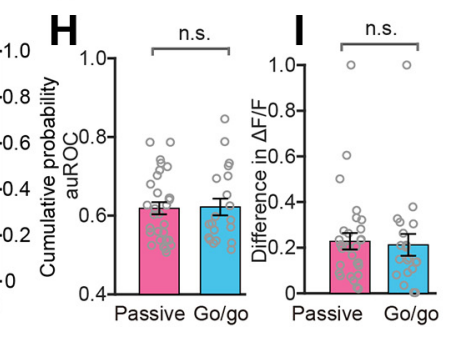

Figure 10. Changes in odor-evoked responses in PPC pyramidal neurons in the passive exposure and go/go task conditions. A, Schema of the experimental paradigm. To record odor-evoked calcium responses of PPC pyramidal neurons, AAV-expressing CaMKII $\alpha$-GCaMP6s was injected into the PPC of C57BL/6J mice. $\boldsymbol{B}$, Expression of GCaMP6s in PPC pyramidal neurons. $\boldsymbol{C}-\boldsymbol{E}$, Heat maps $(\boldsymbol{C}$, trial-averaged traces $(\boldsymbol{D})$, and ROC graph $(\boldsymbol{E})$ of the responses evoked by the two odors under the passive exposure and go/go task conditions. $\boldsymbol{F}$, Averaged traces of odor responses across all animal-odor pairs in the passive exposure ( $n=30$ animal- odor pairs from 10 mice) and go/go task ( $n=20$ animal-odor pairs from 7 mice) conditions. G, Histograms and cumulative probability of auROCs under the passive exposure and go/go task conditions across all animal- odor pairs. $\boldsymbol{H}, \boldsymbol{I}$, Comparison of auROCs $(\boldsymbol{H}) /$ difference in $\Delta F / F(I)$ between the passive exposure and go/go task conditions across all animal- 0 dor pairs. $\boldsymbol{H}$, Unpaired $t$ test, n.s. $(\mathrm{df}=48, t=-0.127)$; (I) Mann-Whitney test, n.s. $(z=-0.287)$.

discriminate odors in a go/no-go task, in which licks to the rewarded odorant are rewarded with water. Thus, task demands, specifically, a requirement to discriminate between odors significantly modulates the odor representation in the $\mathrm{M} / \mathrm{T}$ population. Importantly, the task-demand-dependent neural representation observed in the $\mathrm{OB}$ is also found with a time delay in the PPC but is not found in the APC, indicating that these two higher olfactory centers use different odor representation strategies.

\section{Methodological considerations}

Fiber photometry records GCaMP6s fluorescence that reflects neural activity in a subset of neurons located within the light cone below the end of the fiber (Gunaydin et al., 2014; Guo et al., 2015; Meng et al., 2018). This method has been used extensively in a number of recent studies (Li et al., 2016; Fang et al., 2018; Wei et al., 2018), a subset of which used it for olfactory research (Zhou et al., 2017; Zhang et al., 2019). Compared with in vivo electrophysiological recordings, fiber photometry allows recordings of calcium signals from genetically defined cell types. This is extremely important when we record the neural activity of pyramidal neurons in APC/PPC since spikes from the interneurons in these brain areas are also easily detected by single-unit electrophysiological recordings (Hu et al., 2017). In the OB, although the spikes acquired by electrodes are mainly from the $\mathrm{M} / \mathrm{Ts}$ but not interneurons (Kay and Laurent, 1999; Li et al., 2015, 2017), and the calcium signals recorded by fiber photometry are unable to dif- ferentiate mitral cells from tufted cells, these calcium signals from $\mathrm{M} / \mathrm{T}$ population provide a new method to monitor neural activity from the OB. Our present study characterized the changes in odorevoked calcium fluorescence in the $\mathrm{M} / \mathrm{T}$ population of the $\mathrm{OB}$ and in the pyramidal cell populations of the APC and PPC. Robust and reliable responses were observed in all the mice recorded, and the fluorescence signals changed with the different odors, indicating that this recording technique works well for characterizing odor-evoked neural activity in these cell populations. Furthermore, the task-demand-dependent neural representation identified by fiber photometry in the $\mathrm{M} / \mathrm{Ts}$ population was further supported by single-unit activity and LFP beta oscillations, suggesting that this method produces results comparable to those obtained through electrophysiological recordings. However, simultaneous recordings will be necessary to directly compare how the calcium fluorescence signals relate to the activity of single units, multiple units, and different frequency bands of the LFP; this is an open question for future studies.

\section{Comparison with previous studies at the single-cell level}

Passive exposure, the go/go task, and the go/no-go task represent three typical task demands. In the passive exposure condition, reward is not associated with either of the two odors, and the mice do not need to either detect or discriminate them actively to obtain a reward (no task demand). In the go/go task, the mice only need to detect the occurrence of an odor to obtain a reward; the mice do not need to discriminate the odors (detection task 

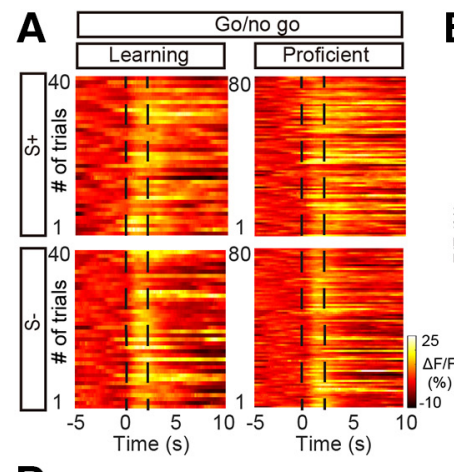

B
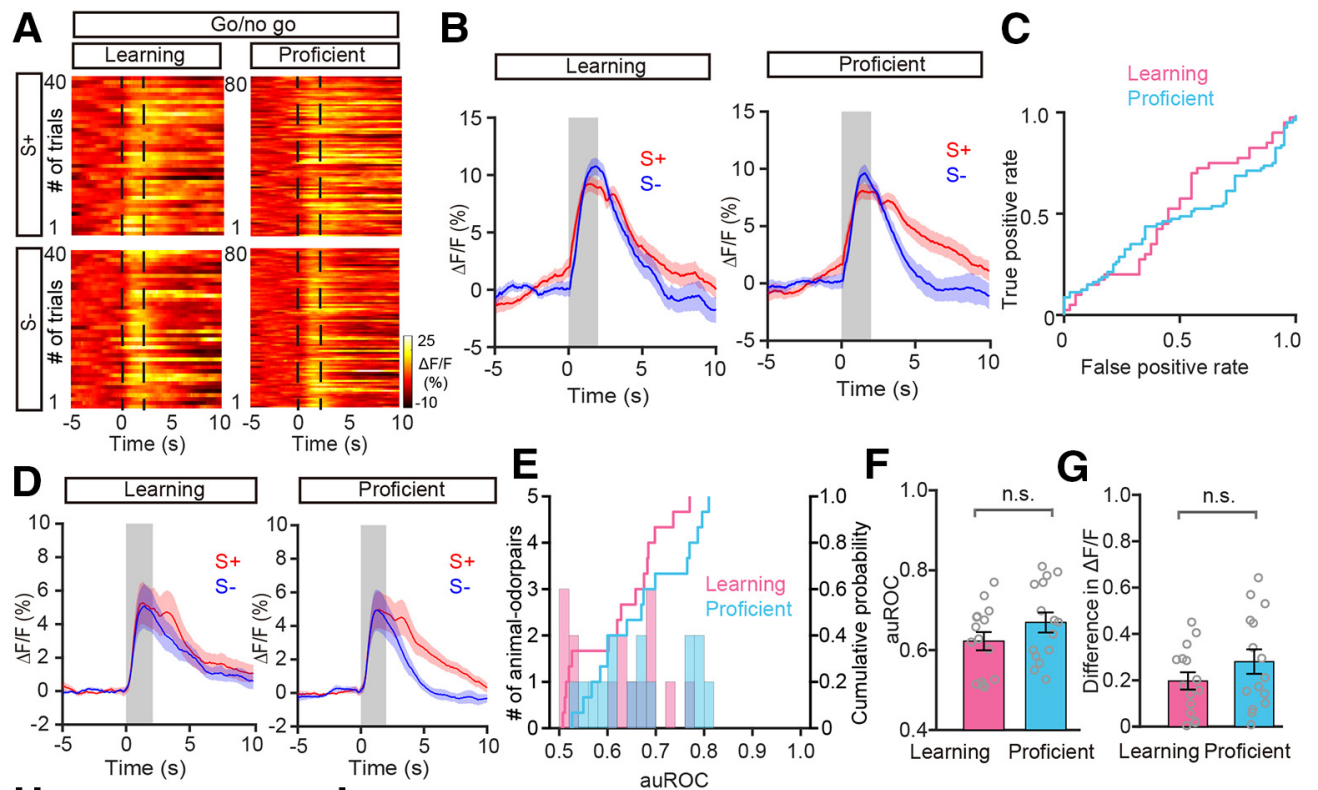

G

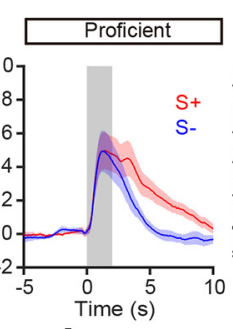

$E^{5}$
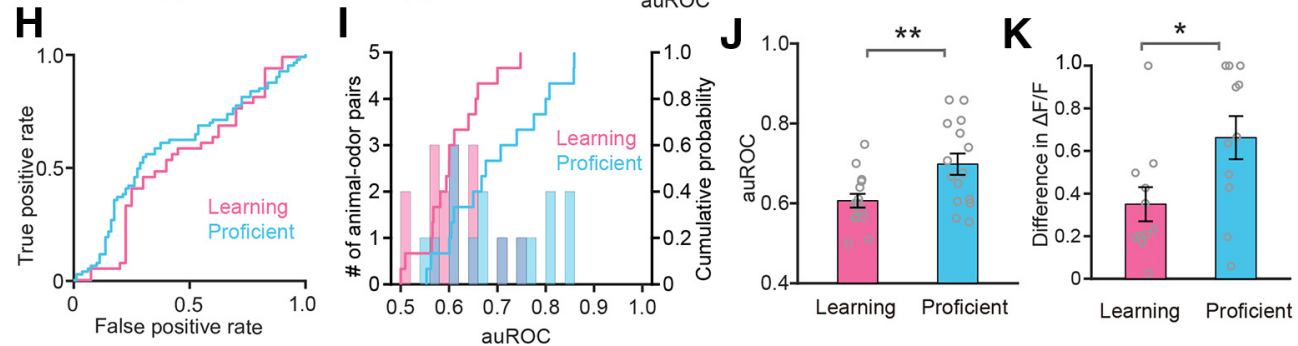

Figure 11. Differentiation of odor responses in PPC pyramidal neurons during the go/no-go task. $\boldsymbol{A}-\boldsymbol{C}$, Heat maps $(\boldsymbol{A})$, trial-averaged traces $(\boldsymbol{B})$, and the ROC graph $(\boldsymbol{C} ; 0-2 \mathrm{~s})$ for the responses evoked by $S+$ and $S-$ in the learning state and in the proficient state during the go/no-go task, from a representative mouse. $D$, Averaged odor-response traces across all animal- odor pairs $(n=$ 15 animal-odor pairs from 7 mice). $\boldsymbol{E}$, Histograms and cumulative probability of auROCs $(0-2 s)$ in the learning state and in the proficient state during the go/no-go task across all animal- odor pairs. $\boldsymbol{F}, \boldsymbol{G}$, Comparison of auROCs $(\boldsymbol{F} ; 0-2 \mathrm{~s}) /$ difference in $\Delta F / F(G ; 0-2 s)$ between the learning state and the proficient state during the go/no-go task across all animal- odor pairs. $\boldsymbol{F}$, Paired $t$ test, n.s. $(\mathrm{df}=14, t=-1.649) ;(\boldsymbol{G})$ paired $t$ test, n.s. (df $=14, t=-1.888)$. $\boldsymbol{H}$, The ROC graph $(4-6 \mathrm{~s})$ for the odor responses shown in $\boldsymbol{B}$. $\boldsymbol{I}$, Histograms and cumulative probability of auROCs $(4-6 \mathrm{~s})$ in the learning state and in the proficient state during the go/no-go task across all animal-odor pairs. J, $\boldsymbol{K}$, Comparison of auROCs $(J, 4-6 \mathrm{~s}) /$ difference in $\Delta F / F(K ; 4-6 \mathrm{~s})$ between the learning state and the proficient state during the go/no-go task across all animal-odor pairs. J, Paired $t$ test, ${ }^{* *} p=0.01(\mathrm{df}=14, t=-3.001) ;(K)$ Wilcoxon's sign rank test, ${ }^{*} p<0.05$ $(z=-2.291)$.

demand). In the go/no-go task, the mice have to discriminate the odors to obtain a reward (discrimination task demand). Previous studies have compared the odor response properties of single M/Ts between passive exposure and active discrimination (Doucette and Restrepo, 2008; Fuentes et al., 2008), as well as between go/go and go/no-go tasks (Li et al., 2015, 2017). However, these studies were not designed to systematically investigate and compare the odor representation strategies underlying these three different tasks. More importantly, our study was designed to address this issue at the M/T cell population level, and parallel experiments were performed in the pyramidal neurons of the APC and PPC. The results from the different experiments consistently point to the conclusion that odor responses of the $\mathrm{M} / \mathrm{T}$ population in the $\mathrm{OB}$ and the pyramidal neuron population in the PPC are dependent on task demands. Odor representation in the $\mathrm{OB}$ is a rather complex process, involving both the firing rate and temporal information (Li et al., 2019). In the present study, we mainly focused on the mean response during the $2 \mathrm{~s}$ odor application, as in a number of previous studies (Doucette and Restrepo, 2008; Li et al., 2015; Yamada et al., 2017). However, other factors, such as the temporal information during and after the odor application, might be also important for the odor representation (Cury and Uchida, 2010). Whether the temporal representation of odor in- formation is dependent on task demands requires further investigation in future studies.

\section{Possible mechanisms underlying the task-demand-dependent neural representation}

The task-demand-dependent neural representation of sensory information is considered a higher cognitive function of the brain since it is a complex process related to learning, experience, and attention. Therefore, this property of neural coding is usually observed in the neurons of associative cortex but not in neural centers below primary cortex (Murakami et al., 2005; Otazu et al., 2009; Wilson and Sullivan, 2011; Carlson et al., 2018). It is therefore somewhat surprising that, after only the first transmission of information from the olfactory periphery, the odor representation of $\mathrm{M} / \mathrm{T}$ s in the $\mathrm{OB}$ is highly dependent on the task demands. However, given the complicated neural circuits within the $\mathrm{OB}$ and between the $\mathrm{OB}$ and other brain centers (Linster and Cleland, 2016; Burton, 2017), our finding is not entirely unexpected. M/Ts receive dense cortical feedback as well as direct and indirect modulatory innervation (through inhibition by local granule cells and other interneurons within the OB; Linster and Cleland, 2016; Burton, 2017). Feedback input from the piriform cortex plays an important role in the representation of odor identification in 

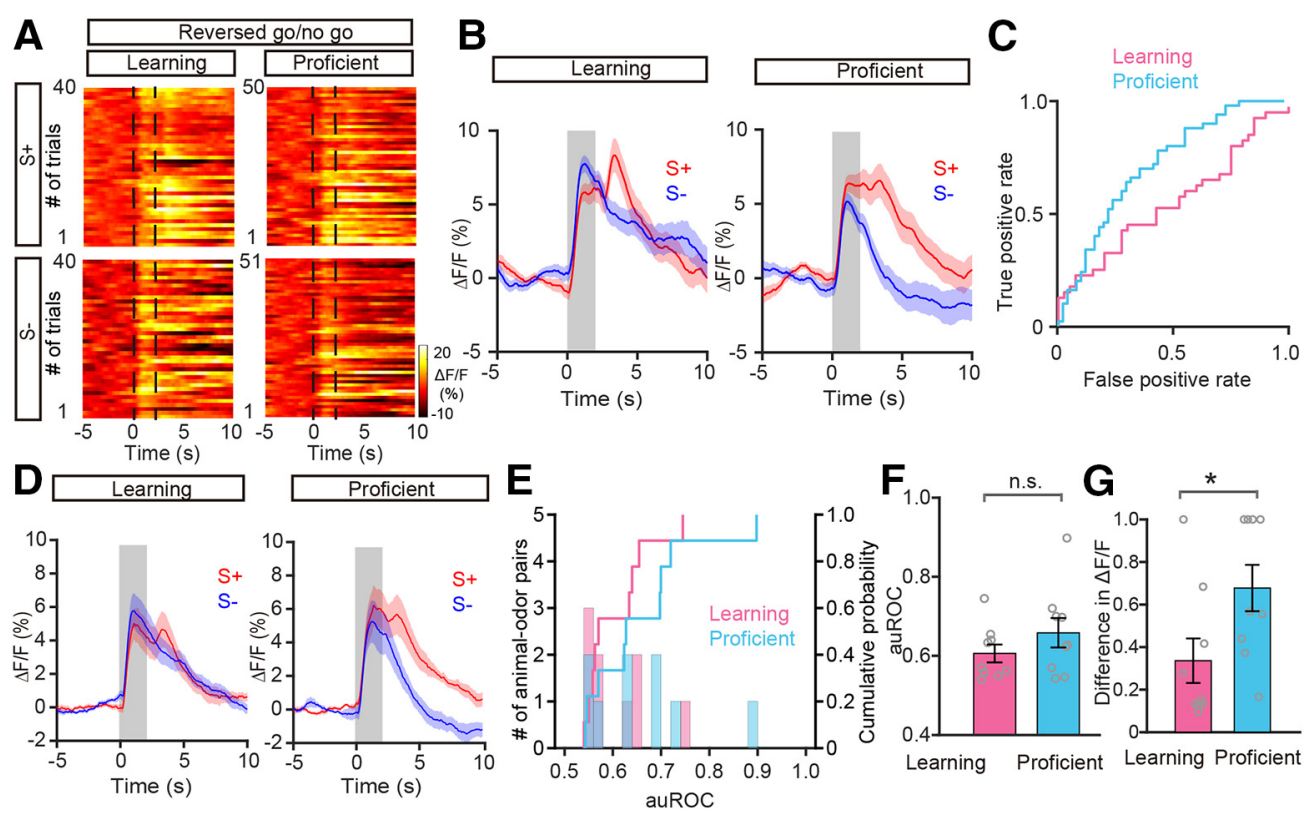

Figure 12. Improved differentiation in the PPC pyramidal neurons in the reversed go/no-go task. $\boldsymbol{A}-\boldsymbol{C}$, Heat maps $(\boldsymbol{A})$, trial-averaged traces $(\boldsymbol{B})$, and ROC graph $(\boldsymbol{C})$ of responses evoked by $S+$ and $S-$ under learning and proficient states during the reversed go/no-go task from a representative mouse. $\boldsymbol{D}$, Averaged odor-response traces across all animal -0 dor pairs $(n=9$ animal- 0 dor pairs from 4 mice). $\boldsymbol{E}$, Histograms and cumulative probability of auROCs in the learning state and in the proficient state during the reversed go/no-go task across all animal- odor pairs. $\boldsymbol{F}, \boldsymbol{G}$, Comparison of auROCs $(\boldsymbol{F}) /$ difference in $\Delta F / F(G)$ between the learning state and the proficient state during the reversed go/no-go task across all animal- 0 dor pairs. $\boldsymbol{F}$, Paired $t$ test, n.s. (df $=8$, $t=1.513) ;(G)$ paired $t$ test, ${ }^{*} p<0.05(\mathrm{df}=8, t=-2.686)$.

mitral cells, as well as in shaping learning-induced neural plasticity and reorganization in mitral cells (Yamada et al., 2017). This feedback may contribute to the neural representation strategy observed in the present study, because learning and neural plasticity are two critical features of this strategy.

Neuromodulatory inputs to the $\mathrm{OB}$ may be another important underlying mechanism (Linster and Cleland, 2016). During the go/no-go task, both LFP oscillations and synchronized M/Ts firing carry information about odor value, similar to the results from our present study that the $\mathrm{M} / \mathrm{T}$ population responds to the rewarded odor robustly regardless of odor identity (Doucette et al., 2011; Li et al., 2015; Ramirez-Gordillo et al., 2018). Since noradrenergic input has previously been identified as a key factor in this M/T representation of odor value (Doucette et al., 2011), it seems likely that it may contribute to the task-dependent neural representation reported in this study. Additionally, a recent study found that serotonergic neurons also carry information about stimulus valence, including rewarded odors (Liu et al., 2014). Given the excitatory effect of the serotonergic input to M/Ts (Brunert et al., 2016; Kapoor et al., 2016), it is likely that the divergent responses we observed in $\mathrm{M} / \mathrm{Ts}$, and especially the strong responses to rewarded odors, are shaped by serotonergic input. However, direct evidence for this hypothesis is currently lacking. Future studies could test this by combining electrophysiological recordings with fiber photometry calcium imaging of the serotonergic axons projecting to the $\mathrm{OB}$ in behaving animals. Our results represent a first step toward understanding the synaptic and circuit mechanisms underlying the task-demanddependent neural representation, but additional work will be necessary to understand the precise circuitry involved in this representational strategy.

\section{Comparison between the $\mathrm{OB}$ and $\mathrm{APC} / \mathrm{PPC}$}

In the $\mathrm{OB}$, the glomeruli receive direct inputs from olfactory sensory neurons and carry information about odor identity, as revealed by several different imaging techniques including fMRI and optical imaging (Mori and Sakano, 2011). However, after signal transmission from the olfactory sensory neurons to the $\mathrm{M} / \mathrm{Ts}$ in the $\mathrm{OB}$, odor identity information is retained but appears to be mainly represented by the temporal pattern of activity rather than the mean firing rate, and the capacity to represent odor value emerges (Li et al., 2019). Therefore, in awake behaving rodents, odor value may be represented by the total odor-evoked neural activity in $\mathrm{M} / \mathrm{Ts}$ but odor identity is mainly represented by the coherence between LFP gamma oscillations and spikes (Li et al., 2015), or by the temporal information contained within a sniffing cycle (Cury and Uchida, 2010). The task-demanddependent neural representation in the $\mathrm{OB} \mathrm{M} / \mathrm{Ts}$ identified in our present study provides new information about how the $\mathrm{OB}$ functions under different brain states. Interestingly, the odor representation strategy found in the $\mathrm{M} / \mathrm{T}$ population was not observed in the pyramidal neurons of the APC, a higher olfactory center that receives the direct projections from M/Ts (Igarashi et al., 2012). This indicates that the OB and the APC use different odor representation strategies. This is consistent with previous studies showing that odor identity is encoded in different ways by the OB and APC: whereas M/Ts use temporal coding for odor identity, APC neurons use rate coding (Miura et al., 2012; Uchida et al., 2014). Furthermore, our result is also partly supported by previous studies showing that although neurons in the APC carry a small degree of odor associative information in behaving rats, neurons in the PPC carry a high degree of associative information and are more likely to alter their responses to odor-outcome associations in a task (Calu et al., 2007; Roesch et al., 2007). Therefore, the APC may be an important center for the representation of odor identity (rather than odor value) at the cell population level, even though previous electrophysiological studies have found that, at the single cell level, neurons in the APC can carry information on both odor identity and odor value (Gire et al., 2013a). The discrepancy between the previous electrophysio- 
logical results and the present fiber photometry results is likely due to the different signal properties of the two methods: whereas electrophysiological techniques record electrical signals with high temporal resolution at the single cell level, fiber photometry records calcium signals with relatively slow temporal resolution at the cell population level.

The lack of a task-demand-dependent neural representation of odor information in the APC was somewhat unexpected, given the input to this area from the OB. However, pyramidal neurons in the APC have three major sources of input: the afferent sensory input relayed from the $\mathrm{OB}$, recurrent collaterals from local principal neurons, and top-down innervation from other brain regions (Wilson and Sullivan, 2011; Bolding and Franks, 2018). It has been reported that odor-evoked responses in the APC are dominated by recruitment of intracortical input, rather than $\mathrm{OB}$ input (Poo and Isaacson, 2011). Therefore, the recurrent input and top-down innervation may contribute to the absence of a task-demand-dependent representation of odor information in the APC. Although the task demand information carried by the $\mathrm{M} / \mathrm{Ts}$ is not found in the APC, it was found in the PPC, which also receives direct input from the $\mathrm{M} / \mathrm{Ts}$, especially the mitral cells, and has odor-value-related but not odor-identity-related responses (Calu et al., 2007; Roesch et al., 2007; Wilson and Sullivan, 2011; Igarashi et al., 2012). Interestingly, although the task-demanddependent divergent response appears during the odor application period in the $\mathrm{OB}$, the divergent response is much later in the PPC, appearing after odor removal. This indicates that the divergent responses in the $\mathrm{OB}$ and $\mathrm{PPC}$ may play different roles in the representation of odor information. Because sensory representation can occur both during and after a stimulus, as has been reported in the olfactory system (Patterson et al., 2013), it is not unlikely that the delayed change in odor responses in the PPC plays an important role in odor representation. The fast divergent responses in the $\mathrm{OB}$ could opt for the neural information processing in the higher decision centers by routing differently processed signals to them (Otazu et al., 2009), enabling rapid and precise selection of the appropriate action. On the other hand, the slower divergent responses in the PPC are likely involved in assessment of odor value and may provide important information for further neural processing in the higher brain centers that encode stimulus salience (Uddin, 2015).

In summary, the present findings broaden our understanding of plasticity in M/Ts in awake, behaving mice by showing that odor representation in the M/T population is highly dependent on task demands. This representational strategy, which is also found in the pyramidal neurons of the PPC but not the APC, is likely important for facilitating information processing in higher brain centers associated with decision making and encoding of salience.

\section{References}

Abraham NM, Vincis R, Lagier S, Rodriguez I, Carleton A (2014) Long term functional plasticity of sensory inputs mediated by olfactory learning. eLife 3:e02109.

Arenkiel BR, Peca J, Davison IG, Feliciano C, Deisseroth K, Augustine GJ, Ehlers MD, Feng G (2007) In vivo light-induced activation of neural circuitry in transgenic mice expressing channelrhodopsin-2. Neuron 54:205-218.

Bao X, Raguet LL, Cole SM, Howard JD, Gottfried J (2016) The role of piriform associative connections in odor categorization. eLife 5:e13732.

Berridge KC, Aldridge JW (2008) Decision utility, the brain, and pursuit of hedonic goals. Soc Cogn 26:621-646.

Bolding KA, Franks KM (2017) Complementary codes for odor identity and intensity in olfactory cortex. eLife 6:e22630.
Bolding KA, Franks KM (2018) Recurrent cortical circuits implement concentration-invariant odor coding. Science 361:eaat6904.

Brunert D, Tsuno Y, Rothermel M, Shipley MT, Wachowiak M (2016) Celltype-specific modulation of sensory responses in olfactory bulb circuits by serotonergic projections from the raphe nuclei. J Neurosci 36:6820-6835.

Burton SD (2017) Inhibitory circuits of the mammalian main olfactory bulb. J Neurophysiol 118:2034-2051.

Buzsáki G, Anastassiou CA, Koch C (2012) The origin of extracellular fields and currents: EEG, ECoG, LFP and spikes. Nat Rev Neurosci 13:407-420.

Calu DJ, Roesch MR, Stalnaker TA, Schoenbaum G (2007) Associative encoding in posterior piriform cortex during odor discrimination and reversal learning. Cereb Cortex 17:1342-1349.

Carlson KS, Gadziola MA, Dauster ES, Wesson DW (2018) Selective attention controls olfactory decisions and the neural encoding of odors. Curr Biol 28:2195-2205.e4.

Chong E, Rinberg D (2018) Behavioral readout of spatio-temporal codes in olfaction. Curr Opin Neurobiol 52:18-24.

Chu MW, Li WL, Komiyama T (2016) Balancing the robustness and efficiency of odor representations during learning. Neuron 92:174-186.

Cury KM, Uchida N (2010) Robust odor coding via inhalation-coupled transient activity in the mammalian olfactory bulb. Neuron 68:570-585.

Doucette W, Restrepo D (2008) Profound context-dependent plasticity of mitral cell responses in olfactory bulb. PLoS Biol 6:e258.

Doucette W, Gire DH, Whitesell J, Carmean V, Lucero MT, Restrepo D (2011) Associative cortex features in the first olfactory brain relay station. Neuron 69:1176-1187.

Economo MN, Hansen KR, Wachowiak M (2016) Control of mitral/tufted cell output by selective inhibition among olfactory bulb glomeruli. Neuron 91:397-411.

Fang YY, Yamaguchi T, Song SC, Tritsch NX, Lin D (2018) A hypothalamic midbrain pathway essential for driving maternal behaviors. Neuron 98: 192-207.e10.

Fawcett T (2006) An introduction to ROC analysis. Pattern Recognit Lett 27:861-874.

Fontanini A, Katz DB (2008) Behavioral states, network states, and sensory response variability. J Neurophysiol 100:1160-1168.

Frederick DE, Brown A, Brim E, Mehta N, Vujovic M, Kay LM (2016) Gamma and beta oscillations define a sequence of neurocognitive modes present in odor processing. J Neurosci 36:7750-7767.

Fuentes RA, Aguilar MI, Aylwin ML, Maldonado PE (2008) Neuronal activity of mitral-tufted cells in awake rats during passive and active odorant stimulation. J Neurophysiol 100:422-430.

Gire DH, Whitesell JD, Doucette W, Restrepo D (2013a) Information for decision-making and stimulus identification is multiplexed in sensory cortex. Nat Neurosci 16:991-993.

Gire DH, Restrepo D, Sejnowski TJ, Greer C, De Carlos JA, LopezMascaraque L (2013b) Temporal processing in the olfactory system: can we see a smell? Neuron 78:416-432.

Gschwend O, Abraham NM, Lagier S, Begnaud F, Rodriguez I, Carleton A (2015) Neuronal pattern separation in the olfactory bulb improves odor discrimination learning. Nat Neurosci 18:1474-1482.

Gunaydin LA, Grosenick L, Finkelstein JC, Kauvar IV, Fenno LE, Adhikari A, Lammel S, Mirzabekov JJ, Airan RD, Zalocusky KA, Tye KM, Anikeeva P, Malenka RC, Deisseroth K (2014) Natural neural projection dynamics underlying social behavior. Cell 157:1535-1551.

Guo QC, Zhou JF, Feng QR, Lin R, Gong H, Luo QM, Zeng SQ, Luo MM, Fu L (2015) Multi-channel fiber photometry for population neuronal activity recording. Biomed Opt Express 6:3919-3931.

Gutnisky DA, Beaman C, Lew SE, Dragoi V (2017) Cortical response states for enhanced sensory discrimination. eLife 6:e29226.

Hu R, Zhang J, Luo M, Hu J (2017) Response patterns of GABAergic neurons in the anterior piriform cortex of awake mice. Cereb Cortex 27: $3110-3124$.

Igarashi KM, Ieki N, An M, Yamaguchi Y, Nagayama S, Kobayakawa K, Kobayakawa R, Tanifuji M, Sakano H, Chen WR, Mori K (2012) Parallel mitral and tufted cell pathways route distinct odor information to different targets in the olfactory cortex. J Neurosci 32:7970-7985.

Jeanne JM, Sharpee TO, Gentner TQ (2013) Associative learning enhances population coding by inverting interneuronal correlation patterns. Neuron 78:352-363.

Jiang H, Schuele S, Rosenow J, Zelano C, Parvizi J, Tao JX, Wu S, Gottfried JA 
(2017) Theta oscillations rapidly convey odor-specific content in human piriform cortex. Neuron 94:207-219.e4.

Jordan R, Fukunaga I, Kollo M, Schaefer AT (2018) Active sampling state dynamically enhances olfactory bulb odor representation. Neuron 98:12141228.e5.

Kapoor V, Provost AC, Agarwal P, Murthy VN (2016) Activation of raphe nuclei triggers rapid and distinct effects on parallel olfactory bulb output channels. Nat Neurosci 19:271-282.

Kato HK, Chu MW, Isaacson JS, Komiyama T (2012) Dynamic sensory representations in the olfactory bulb: modulation by wakefulness and experience. Neuron 76:962-975.

Kay LM (2015) Olfactory system oscillations across phyla. Curr Opin Neurobiol 31:141-147.

Kay LM, Laurent G (1999) Odor- and context-dependent modulation of mitral cell activity in behaving rats. Nat Neurosci 2:1003-1009.

Kikuta S, Fletcher ML, Homma R, Yamasoba T, Nagayama S (2013) Odorant response properties of individual neurons in an olfactory glomerular module. Neuron 77:1122-1135.

Koldaeva A, Schaefer AT, Fukunaga I (2019) Rapid task-dependent tuning of the mouse olfactory bulb. eLife 8:e43558.

Li A, Gong L, Xu F (2011) Brain-state-independent neural representation of peripheral stimulation in rat olfactory bulb. Proc Natl Acad Sci U S A 108:5087-5092.

Li A, Gire DH, Bozza T, Restrepo D (2014) Precise detection of direct glomerular input duration by the olfactory bulb. J Neurosci 34:16058-16064.

Li A, Gire DH, Restrepo D (2015) Upsilon spike-field coherence in a population of olfactory bulb neurons differentiates between odors irrespective of associated outcome. J Neurosci 35:5808-5822.

Li A, Guthman EM, Doucette WT, Restrepo D (2017) Behavioral status influences the dependence of odorant-induced change in firing on prestimulus firing rate. J Neurosci 37:1835-1852.

Li A, Rao X, Zhou Y, Restrepo D (2019) Complex neural representation of odour information in the olfactory bulb. Acta Physiol 2019:e13333.

Li Y, Zhong W, Wang D, Feng Q, Liu Z, Zhou J, Jia C, Hu F, Zeng J, Guo Q, Fu L, Luo M (2016) Serotonin neurons in the dorsal raphe nucleus encode reward signals. Nat Commun 7:10503.

Linster C, Cleland TA (2016) Neuromodulation of olfactory transformations. Curr Opin Neurobiol 40:170-177.

Liu Z, Zhou J, Li Y, Hu F, Lu Y, Ma M, Feng Q, Zhang JE, Wang D, Zeng J, Bao J, Kim JY, Chen ZF, El Mestikawy S, Luo M (2014) Dorsal raphe neurons signal reward through 5-HT and glutamate. Neuron 81:1360-1374.

Martin C, Ravel N (2014) Beta and gamma oscillatory activities associated with olfactory memory tasks: different rhythms for different functional networks? Front Behav Neurosci 8:218.

Meissner-Bernard C, Dembitskaya Y, Venance L, Fleischmann A (2019) Encoding of odor fear memories in the mouse olfactory cortex. Curr Biol 29:367-380.e4.

Meng CB, Zhou JH, Papaneri A, Peddada T, Xu KR, Cui GH (2018) Spectrally resolved fiber photometry for multi-component analysis of brain circuits. Neuron 98:707-717.e4.

Miura K, Mainen ZF, Uchida N (2012) Odor representations in olfactory cortex: distributed rate coding and decorrelated population activity. Neuron 74:1087-1098.

Mori K, Sakano H (2011) How is the olfactory map formed and interpreted in the mammalian brain? Annu Rev Neurosci 34:467-499.

Murakami M, Kashiwadani H, Kirino Y, Mori K (2005) State-dependent sensory gating in olfactory cortex. Neuron 46:285-296.

Nunez-Parra A, Li A, Restrepo D (2014) Coding odor identity and odor value in awake rodents. Prog Brain Res 208:205-222.
Otazu GH, Tai LH, Yang Y, Zador AM (2009) Engaging in an auditory task suppresses responses in auditory cortex. Nat Neurosci 12:646-654.

Padmanabhan K, Osakada F, Tarabrina A, Kizer E, Callaway EM, Gage FH, Sejnowski TJ (2016) Diverse representations of olfactory information in centrifugal feedback projections. J Neurosci 36:7535-7545.

Patterson MA, Lagier S, Carleton A (2013) Odor representations in the olfactory bulb evolve after the first breath and persist as an odor afterimage. Proc Natl Acad Sci U S A 110:E3340-E3349.

Pesaran B, Vinck M, Einevoll GT, Sirota A, Fries P, Siegel M, Truccolo W, Schroeder CE, Srinivasan R (2018) Investigating large-scale brain dynamics using field potential recordings: analysis and interpretation. Nat Neurosci 21:903-919.

Poo C, Isaacson JS (2011) A major role for intracortical circuits in the strength and tuning of odor-evoked excitation in olfactory cortex. Neuron $72: 41-48$

Ramirez-Gordillo D, Ma M, Restrepo D (2018) Precision of classification of odorant value by the power of olfactory bulb oscillations is altered by optogenetic silencing of local adrenergic innervation. Front Cell Neurosci $12: 48$

Rinberg D, Koulakov A, Gelperin A (2006) Sparse odor coding in awake behaving mice. J Neurosci 26:8857-8865.

Roesch MR, Stalnaker TA, Schoenbaum G (2007) Associative encoding in anterior piriform cortex versus orbitofrontal cortex during odor discrimination and reversal learning. Cereb Cortex 17:643-652.

Schaffer ES, Stettler DD, Kato D, Choi GB, Axel R, Abbott LF (2018) Odor perception on the two sides of the brain: consistency despite randomness. Neuron 98:736-742.e3.

Sun C, Tang K, Wu J, Xu H, Zhang W, Cao T, Zhou Y, Yu T, Li A (2019) Leptin modulates olfactory discrimination and neural activity in the olfactory bulb. Acta Physiol 227:e13319.

Uchida N, Poo C, Haddad R (2014) Coding and transformations in the olfactory system. Annu Rev Neurosci 37:363-385.

Uddin LQ (2015) Salience processing and insular cortical function and dysfunction. Nat Rev Neurosci 16:55-61.

Vaaga CE, Westbrook GL (2016) Parallel processing of afferent olfactory sensory information. J Physiol 594:6715-6732.

Vaaga CE, Yorgason JT, Williams JT, Westbrook GL (2017) Presynaptic gain control by endogenous cotransmission of dopamine and GABA in the olfactory bulb. J Neurophysiol 117:1163-1170.

Wachowiak M, Economo MN, Díaz-Quesada M, Brunert D, Wesson DW, White JA, Rothermel M (2013) Optical dissection of odor information processing in vivo using GCaMPs expressed in specified cell types of the olfactory bulb. J Neurosci 33:5285-5300.

Wei C, Han X, Weng D, Feng Q, Qi X, Li J, Luo M (2018) Response dynamics of midbrain dopamine neurons and serotonin neurons to heroin, nicotine, cocaine, and MDMA. Cell Discov 4:60.

Wilson CD, Serrano GO, Koulakov AA, Rinberg D (2017) A primacy code for odor identity. Nat Commun 8:1477.

Wilson DA, Sullivan RM (2011) Cortical processing of odor objects. Neuron 72:506-519.

Yamada Y, Bhaukaurally K, Madarász TJ, Pouget A, Rodriguez I, Carleton A (2017) Context- and output layer-dependent long-term ensemble plasticity in a sensory circuit. Neuron 93:1198-1212.e5.

Zhang W, Sun C, Shao Y, Zhou Z, Hou Y, Li A (2019) Partial depletion of dopaminergic neurons in the substantia nigra impairs olfaction and alters neural activity in the olfactory bulb. Sci Rep 9:254.

Zhou Y, Wang X, Cao T, Xu J, Wang D, Restrepo D, Li A (2017) Insulin modulates neural activity of pyramidal neurons in the anterior piriform cortex. Front Cell Neurosci 11:378. 